\title{
Article \\ Defining Phenotype, Tropism, and Retinal Gene Therapy Using Adeno-Associated Viral Vectors (AAVs) in New-Born Brown Norway Rats with a Spontaneous Mutation in Crb1
}

\author{
Nanda Boon ${ }^{1}{ }^{(}$, , C. Henrique Alves ${ }^{1}{ }^{\circledR}$, Aat A. Mulder ${ }^{2}$, Charlotte A. Andriessen ${ }^{1}$, Thilo M. Buck ${ }^{1}(\mathbb{D}$, \\ Peter M. J. Quinn $\left.{ }^{1}{ }^{(}\right)$, Rogier M. Vos ${ }^{1}$, Abraham J. Koster ${ }^{2}$, Carolina R. Jost ${ }^{2}{ }^{\circledR}$ and Jan Wijnholds ${ }^{1,3, *(1)}$ \\ 1 Department of Ophthalmology, Leiden University Medical Center (LUMC), Albinusdreef 2, \\ 2333 ZA Leiden, The Netherlands; n.boon@lumc.nl (N.B.); chalves@fmed.uc.pt (C.H.A.); \\ C.A.Andriessen@lumc.nl (C.A.A.); T.M.Buck@lumc.nl (T.M.B.); pq2138@cumc.columbia.edu (P.M.J.Q.); \\ rogier_vos@hotmail.com (R.M.V.) \\ 2 Department of Cell \& Chemical Biology, Leiden University Medical Center (LUMC), \\ 2300 RC Leiden, The Netherlands; A.A.Mulder@lumc.nl (A.A.M.); A.J.Koster@lumc.nl (A.J.K.); \\ C.R.Jost@lumc.nl (C.R.J.) \\ 3 Netherlands Institute for Neuroscience, Institute of the Royal Netherlands Academy of Arts and \\ Sciences (KNAW), Meibergdreef 47, 1105 BA Amsterdam, The Netherlands \\ * Correspondence: j.wijnholds@lumc.nl
}

Citation: Boon, N.; Alves, C.H.; Mulder, A.A.; Andriessen, C.A.; Buck, T.M.; Quinn, P.M.J.; Vos, R.M.; Koster, A.J.; Jost, C.R.; Wijnholds, J. Defining Phenotype, Tropism, and Retinal Gene Therapy Using

Adeno-Associated Viral Vectors (AAVs) in New-Born Brown Norway Rats with a Spontaneous Mutation in Crb1. Int. J. Mol. Sci. 2021, 22, 3563. https://doi.org/10.3390/ijms22073563

Academic Editor:

J. Fielding Hejtmancik

Received: 25 February 2021

Accepted: 26 March 2021

Published: 30 March 2021

Publisher's Note: MDPI stays neutral with regard to jurisdictional claims in published maps and institutional affiliations.

Copyright: () 2021 by the authors. Licensee MDPI, Basel, Switzerland. This article is an open access article distributed under the terms and conditions of the Creative Commons Attribution (CC BY) license (https:// creativecommons.org/licenses/by/ $4.0 /)$.
Abstract: Mutations in the Crumbs homologue 1 (CRB1) gene cause inherited retinal dystrophies, such as early-onset retinitis pigmentosa and Leber congenital amaurosis. A Brown Norway rat strain was reported with a spontaneous insertion-deletion (indel) mutation in exon 6 of Crb1. It has been reported that these $\mathrm{Crb1}$ mutant rats show vascular abnormalities associated with retinal telangiectasia and possess an early-onset retinal degenerative phenotype with outer limiting membrane breaks and focal loss of retinal lamination at 2 months of age. Here, we further characterized the morphological phenotype of new-born and adult Crb1 mutant rats in comparison with age-matched Brown Norway rats without a mutation in Crb1. A significantly decreased retinal function and visual acuity was observed in $\mathrm{Crb} 1$ mutant rats at 1 and 3 months of age, respectively. Moreover, in control rats, the subcellular localization of canonical CRB1 was observed at the subapical region in Müller glial cells while CRB2 was observed at the subapical region in both photoreceptors and Müller glial cells by immuno-electron microscopy. CRB1 localization was lost in the Crb1 mutant rats, whereas CRB2 was still observed. In addition, we determined the tropism of subretinal or intravitreally administered AAV5-, AAV9- or AAV6-variant ShH10 ${ }^{\mathrm{Y} 45 \mathrm{~F}}$ vectors in new-born control and Crb1 mutant rat retinas. We showed that subretinal injection of AAV5 and AAV9 at postnatal days 5 (P5) or 8 (P8) predominantly infected the retinal pigment epithelium (RPE) and photoreceptor cells; while intravitreal injection of $\mathrm{ShH} 10^{\mathrm{Y} 445 \mathrm{~F}}$ at $\mathrm{P} 5$ or P8 resulted in efficient infection of mainly Müller glial cells. Using knowledge of the subcellular localization of CRB1 and the ability of ShH10 ${ }^{\mathrm{Y} 45 \mathrm{~F}}$ to infect Müller glial cells, canonical hCRB1 and hCRB2 AAV-mediated gene therapy were explored in new-born $\mathrm{Crb1}$ mutant rats. Enhanced retinal function after gene therapy delivery in the $\mathrm{Cr} b 1$ rat was not observed. No timely rescue of the retinal phenotype was observed using retinal function and visual acuity, suggesting the need for earlier onset of expression of recombinant hCRB proteins in Müller glial cells to rescue the severe retinal phenotype in Crb1 mutant rats.

Keywords: CRB1; CRB2; Crumbs homologue 1; retinitis pigmentosa; AAV tropism; AAV-mediated gene therapy

\section{Introduction}

Retinitis pigmentosa (RP) and Leber congenital amaurosis (LCA) are inherited retinal degenerative diseases causing progressive vision loss, ultimately leading to blindness. Mutations in the Crumbs homolog 1 (CRB1) gene is a frequent cause of these retinal 
dystrophies in humans [1]. The CRB1 gene, mapped to chromosome 1q31.3, encodes a large transmembrane protein and belongs to the Crumbs (CRB) family, with family members CRB2 and CRB3. CRB proteins are located in the subapical region above adherens junctions at the outer limiting membrane (OLM), where it can interact with, amongst others, PALS1 to form the canonical Crumbs complex [2-4]. $\mathrm{Crb1}$ knockout $\left(\mathrm{Crb} 1^{\mathrm{KO}}\right)$ mouse models show a mild retinal degeneration with OLM disruptions and ectopic rows of photoreceptor cell nuclei in the photoreceptor segment layers from postnatal day 14 (P14) [5]. Concomitant loss of $\mathrm{Crb} 2$ in $\mathrm{Crb} 1^{\mathrm{KO}}$ mice results in a more severe RP or LCA phenotype, depending on which cell type lacks $\mathrm{Cr} b 2$ [6-9]. Currently, there is no treatment available for CRB1-related retinal dystrophies.

A Brown Norway rat strain was described with an inherited retinal degenerative phenotype caused by a spontaneous in frame insertion-deletion (indel) in exon 6 of the Crb1 gene [10]. These Crb1 mutant rats, expressing an alternative CRB1 ${ }^{\mathrm{INDEL}}$ protein, exhibit an early-onset loss of retinal function from 3 weeks of age. In addition, the first signs of retinal degeneration were observed from P15, including OLM disruptions and ectopic rows of photoreceptor cell nuclei in the photoreceptor segment layers. At older ages, these disruptions progress and ultimately lead to a focal loss of retinal lamination [10]. Because of its naturally occurring mutation and early-onset severe retinal phenotype, these rats are a potential attractive animal model for the development of gene therapy. Currently, gene therapy using adeno-associated viral vectors (AAVs) is the leading platform of gene delivery for the treatment of retinal dystrophies because of its low toxicity and ability to target both dividing and non-dividing cells [11]. In addition, different AAV capsids display distinct cell tropisms, making it possible to target different cell types. Moreover, previous mouse gene supplementation studies with AAV expressing human CRB2 (hCRB2) have shown to preserve the retinal morphology and function in Crb1 RP mouse models [12,13]. Altogether, this indicates the potential use of AAV-mediated gene therapy for CRB1-related retinal dystrophies.

Here, we perform a thorough characterization of the morphological phenotype of new-born and adult $\mathrm{Crb1}$ mutant rats and its effect on retinal function and visual acuity in comparison with age-matched control rats. Using immuno-electron-microscopy, we show that, in control rats, canonical CRB1 localizes specifically in Müller glial cells (MGCs) at the subapical region adjacent to the adherens junctions at the outer limiting membrane (OLM), whereas the Crb1 mutant rats barely express detectable levels of CRB1 ${ }^{1 N D E L}$ at the subapical region of MGCs. In turn, CRB2 is localized at the subapical region in MGCs and photoreceptors of both control and $\mathrm{Crb1}$ mutant rats. Next, we describe the tropism of three different AAV serotypes (AAV5-, AAV9- and AAV6-variant ShH10Y445F) expressing GFP driven by the cytomegalovirus (CMV) promoter in new-born control and Crb1 mutant rat retinas. Tropism data at P5 and P8 shows that subretinal injection of AAV5.CMV.GFP and AAV9.CMV.GFP mainly transduces photoreceptors and retinal pigment epithelium (RPE), whereas intravitreal injection at $\mathrm{P} 5$ or $\mathrm{P} 8$ of $\mathrm{ShH} 10^{\mathrm{Y} 445 \mathrm{~F}}$.CMV.GFP efficiently transduces MGCs. Based on this knowledge, AAV-mediated hCRB1 and hCRB2 gene therapy were explored in $\mathrm{P} 5 \mathrm{Crb1}$ mutant rats.

\section{Results}

\subsection{The Spontaneous Mutation in Crb1 in Brown Norway Rats Leads to Retinal Dysfunction and} Vision Impairment

To study the retinal function of the Brown Norway rats with a spontaneous mutation in the Crb1 gene, we performed electroretinography (ERG) in 1-, 3- and 5-month-old Crb1 mutant rats compared to age-matched control Brown Norway rats without a mutation in Crb1. One-month-old Crb1 mutant rats showed significantly reduced a- and b-wave responses under scotopic conditions (Figure $1 \mathrm{~A}, \mathrm{C}$ ). In addition, a significant reduction in photopic b-wave was observed (Figure 1C,D). Finally, there was a significantly reduced flicker amplitude response in $\mathrm{Cr} b 1$ mutant rats compared to age-matched control rats in range $A$ and $B$, indicating aberrations in the rod pathway and cone pathway, respectively (Figure 1E). At 3 and 5 months of age, the retinal degeneration continued in the $\mathrm{Crb1}$ mutant 
rats and the ERG response was further reduced compared to the age-matched control rats (Figure S1).

A
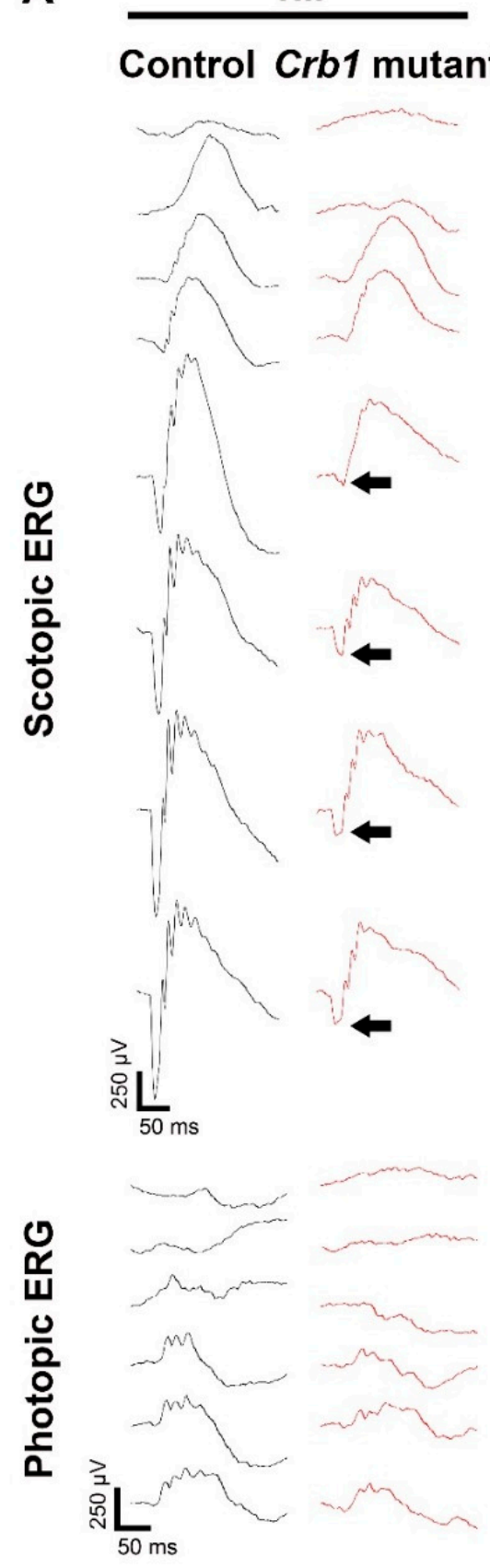

B

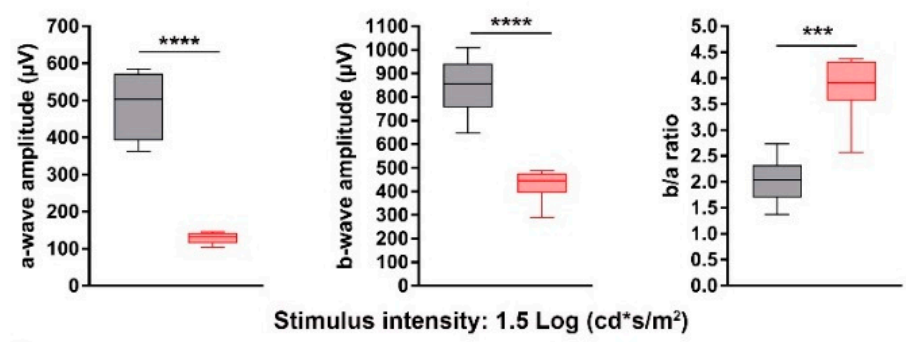

C
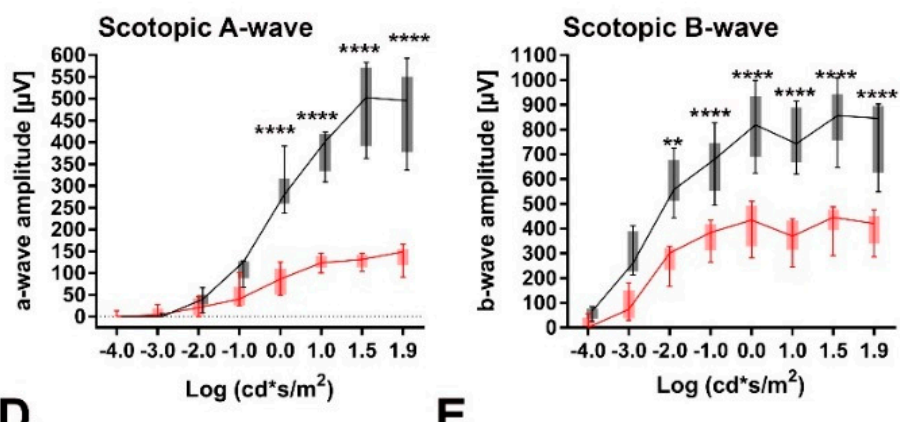

E
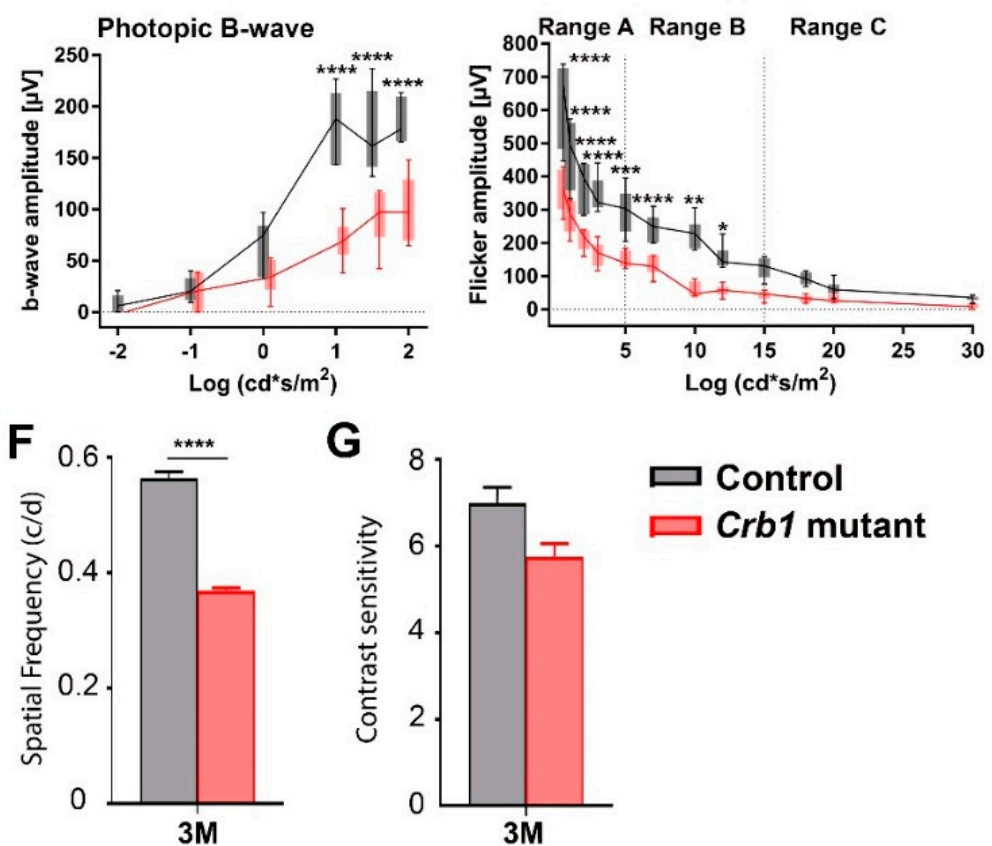

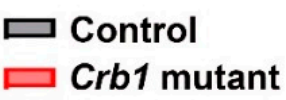

Figure 1. Significantly reduced retinal function in the Crb1 mutant compared to age-matched control rats. (A) Scotopic and photopic single-flash intensity series from representative rats at one month of age. The attenuated scotopic a-wave of the Crb1 mutant rats is indicated with the black arrow. (B) Quantitative analysis of the scotopic a-wave, b-wave and $\mathrm{b}$-wave/a-wave amplitude ratio (b/a ratio). Quantitative analysis of the scotopic a-wave and b-wave (C), the photopic b-wave (D) and the flicker amplitude response (E). Boxes indicate the 25 and $75 \%$ quantile range and whiskers indicate the 5 and $95 \%$ quantiles, and the intersection of line and error bar indicates the median of the data (box-and-whisker plot). Decreased visual function in 3 months of age Crb1 mutant rats measured using the optokinetic head tracking response (OKT) spatial frequency (F) and OKT contrast sensitivity (G). Number of animals used for ERG: $n=6$ for control and Crb1 mutant rats; and for OKT: control $n=4$ and Crb1 mutant $=10$. Mean \pm SEM. ${ }^{*} p<0.05 ;{ }^{* *} p<0.01 ; * * * p<0.001 ;{ }^{* * * *} p<0.0001$. 
In addition, the visual function was determined using an optomotor response test (optokinetic head tracking response (OKT)). The OKT measures spatial frequency threshold, also called visual acuity, by systematically increasing the spatial frequency of the grating at $100 \%$ contrast until the animals no longer perform head tracking. In addition, contrast sensitivity can also be measured, where the minimum contrast that generated a tracking response was identified over a range of spatial frequencies [14,15]. Three-month-old Crb1 mutant rats showed a significantly decreased OKT spatial frequency, indicating a loss in visual function compared to control rats (Figure 1F,G). In summary, Crb1 mutant rats presents a severely decreased retinal function and visual function within three months of age.

\subsection{First Signs of Retinal Degeneration in Crb1 Mutant Rats Are Observed from Postnatal Day 10}

To study the morphological phenotype of new-born Crb1 mutant rats, histological analysis of retina sections was performed and compared to age-matched controls. No abnormalities in the retinal development and lamination were observed in P5 Crb1 mutant rats compared with the control (Figure 2A,E). In addition, immunohistochemical analysis of $\mathrm{P} 5 \mathrm{Crb1}$ mutant and control rat retinas showed similar localization of photoreceptors in the outer nuclear layer (ONL), indicated by recoverin and rhodopsin staining (Figure 2B,F); MGC localization within the inner nuclear layer (INL), indicated by SOX9 staining (Figure 2C,G); and a continuous OLM, indicated by localization of subapical region marker PALS1 and adherens junctions marker P120-catenin (Figure 2D,H). In accordance with previous data [16], glutamine synthetase (GS), a mature MGC marker, was not yet fully expressed in the P5 control nor Crb1 mutant retinas (Figure 2C,G).

In contrast to previous findings, where the phenotype was first observed at P15 [10], here we observed focal disruptions already at P10 in the Crb1 mutant rat retina (Figure 2M). The phenotype includes OLM breaks (Figure 2P, arrowhead) and photoreceptor cell nuclei protrusions into the photoreceptor segment layers (Figure 2N). Interestingly, PALS1 staining is more diffuse in P10 Crb1 mutant rats in comparison to age-matched controls (Figure 2L,P). In addition, at P10, low levels of GS were detectable and appeared similar between control and $\mathrm{Crb} 1$ mutant retinas (Figure 2K,O). One-month-old control rat retinas present mature photoreceptor cells, MGCs with radial processes throughout the retinal layers and a continuous OLM (Figure 2Q-T). In contrast, the retina of Crb1 mutant rats is severely disorganized (Figure $2 \mathrm{U}$ ), characterized by, at the foci, intermingling of nuclei from the INL and ONL and loss of photoreceptor inner and outer segments (Figure 2V, arrowhead), misplaced SOX9-positive MGC nuclei and disorganized radial MGC processes (Figure 2W). In addition, we observed OLM breaks indicated by disruptions in PALS1 and P120-catenin staining (Figure 2X, arrowhead). Similar results were obtained in 2-months-old Crb1 mutant rats, where focal loss of retinal lamination, OLM disruptions and photoreceptor alterations were observed [10]. Altogether, these results highlight the early-onset degenerative retinal phenotype in $\mathrm{Crb1}$ mutant compared to control rats. 


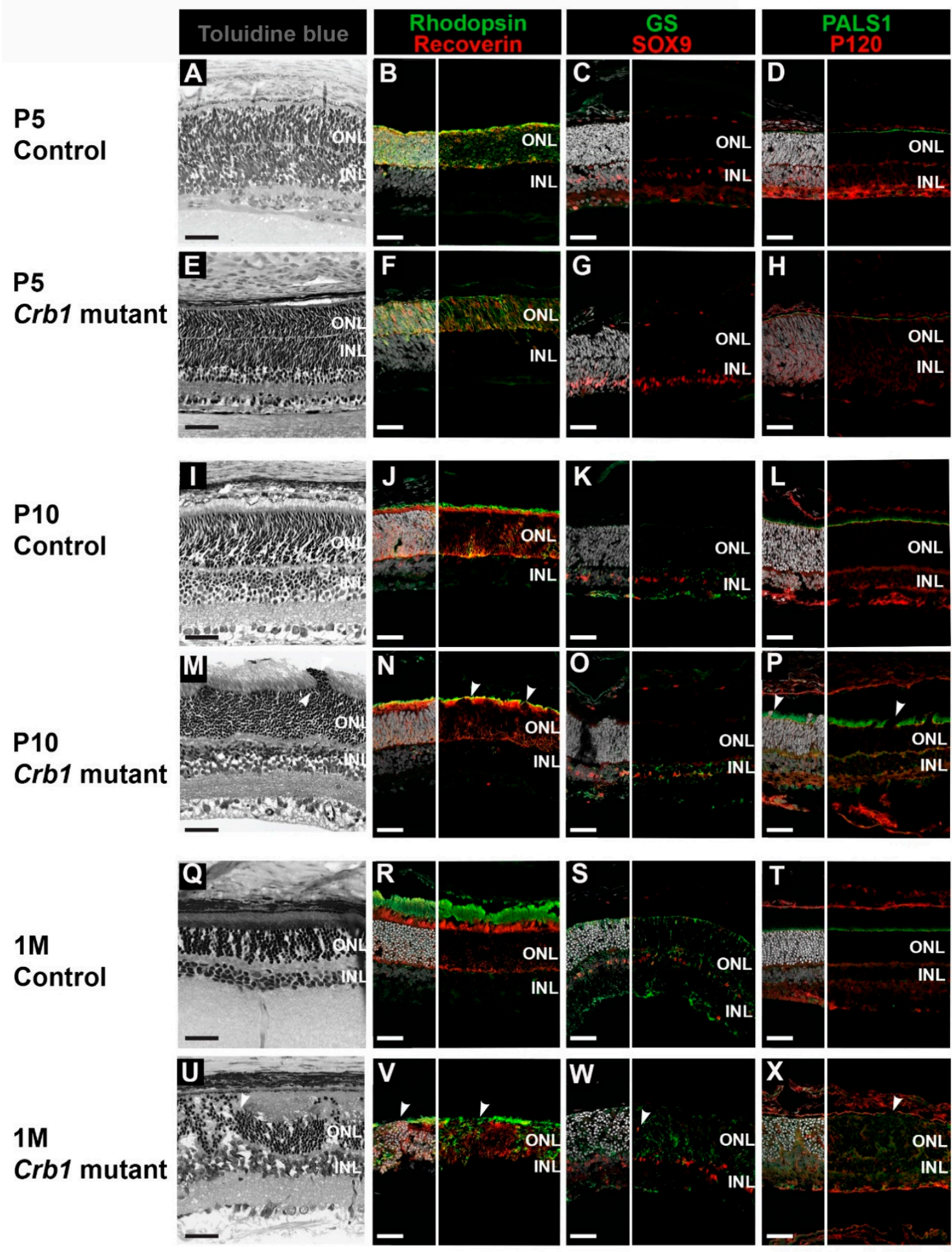

Figure 2. Retinal degeneration is observed from postnatal day 10 retinas of Crb1 mutant rats. Light microscopy of toluidine blue-stained retinal sections from P5, P10 and 1-month-old control (A,I,Q) and Crb1 mutant rats (E,M,U). Immunohistochemistry staining of P5, P10 and 1-month-old retinal sections, with recoverin and rhodopsin $(\mathbf{B}, \mathbf{F}, \mathbf{J}, \mathbf{N}, \mathbf{R}, \mathbf{V})$, glutamine synthetase (GS) and SOX9 (C,G,K,O,S,W) and PALS1 with P120-catenin (D,H,L,P,T,X). No abnormalities were observed in the control retina (A-D,I-L, Q-T). In the Crb1 mutant rats, all retinal layers were formed and properly laminated at P5 (E-H). From P10 onwards, degeneration at the foci was observed in the Crb1 mutant rat retina by photoreceptor nuclei protrusions into the photoreceptor segment layers, misplaced Müller glial cells (MGCs) and disruption of the outer limiting membrane (OLM) (M-P,U-X; arrowheads). At least $n=2$ eyes were used per time point. GCL, ganglion cell layer; INL, inner nuclear layer; ONL, outer nuclear layer; RPE, retinal pigment epithelium. Scale bar: $40 \mu \mathrm{m}$. 


\subsection{The Crb1 Mutant Rat Retina Develops a Progressive Lack of Retinal Lamination}

Using SD-OCT imaging, the retinal degeneration was followed in vivo at P17 and 1, 2 and 3 months of age $\mathrm{Crb1}$ mutant rats in comparison with control rats. All retinal layers were correctly laminated at all ages measured in the control rat (Figure $\left.3 A^{\prime}, C^{\prime}, E^{\prime}, G^{\prime}\right)$. At $\mathrm{P} 17$, retinal lamination in $\mathrm{Crb1}$ mutant rats appeared similar to control rats (Figure 3B'). At one-month-old Crb1 mutant rats, the retinal lamination is affected mainly at the OLM indicated by a hyperreflective ONL (Figure 3D', arrowhead). Two and three months of age $\mathrm{Crb1}$ mutant rats show a further increased retinal degeneration, indicated by a hyperreflective INL and ONL, resulting in an unclear distinction between the INL and the ONL (Figure $3 \mathrm{~F}^{\prime}, \mathrm{H}^{\prime}$, arrowhead). Volume intensity projection (VIP) shows disruptions throughout the $\mathrm{Cr} b 1$ mutant retina (Figure 3B,D,F,H). Morphological sections of the Crb1 mutant rats show a similar degeneration, with disruptions at the OLM and intermingling of nuclei of the INL and ONL at 1 and 3 months of age rats (Figure 2M,Q,U).

\section{Control}

P17

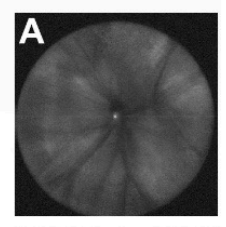

1M

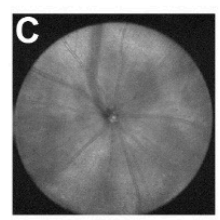

$2 \mathrm{M}$
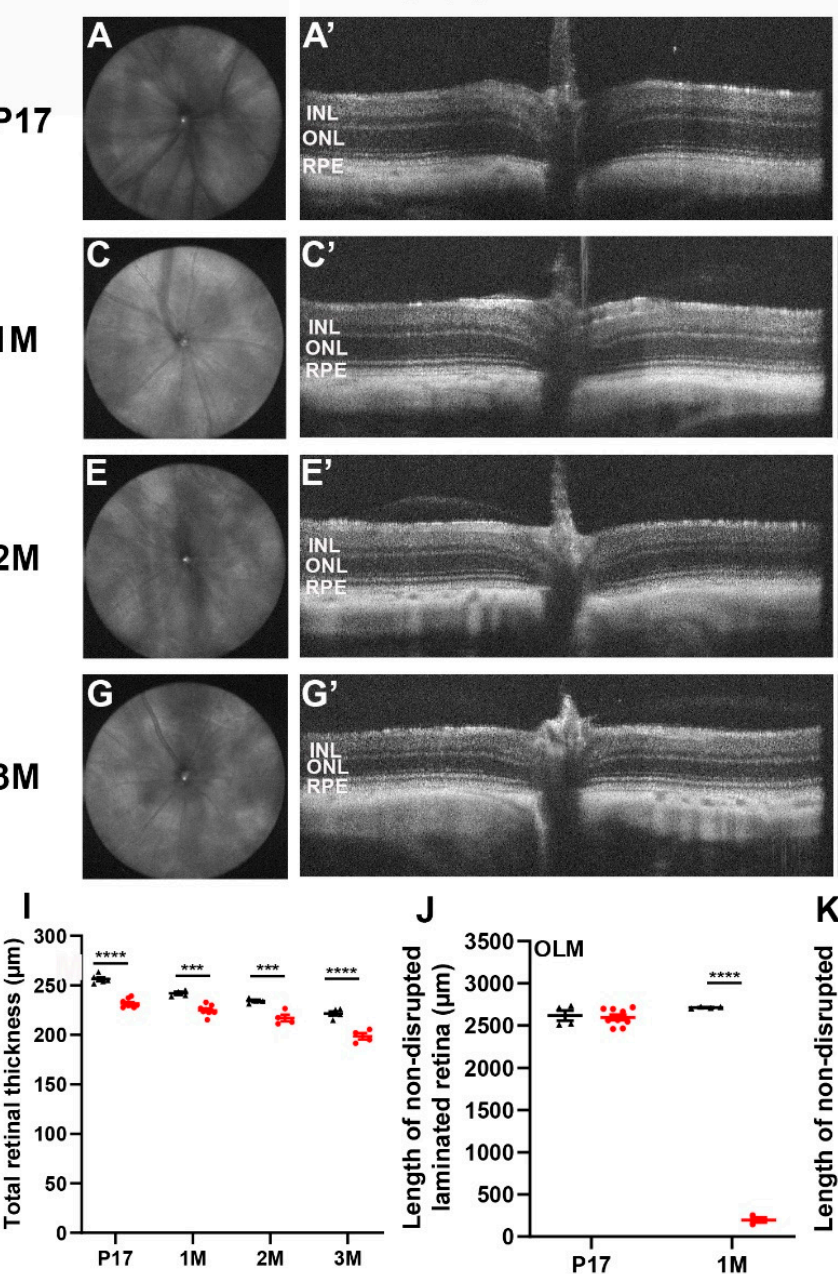

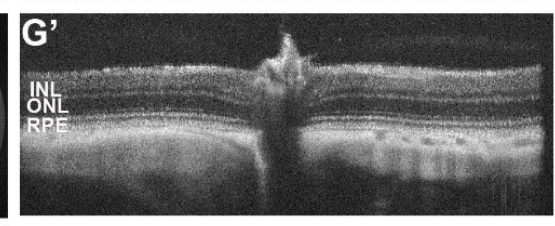

J

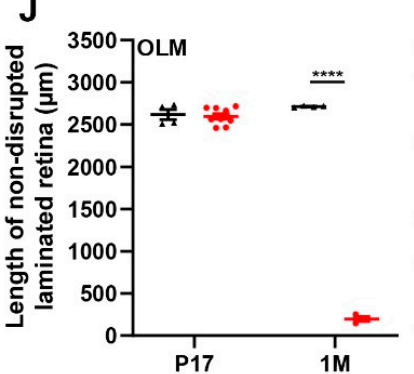

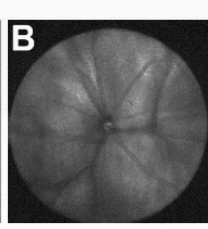
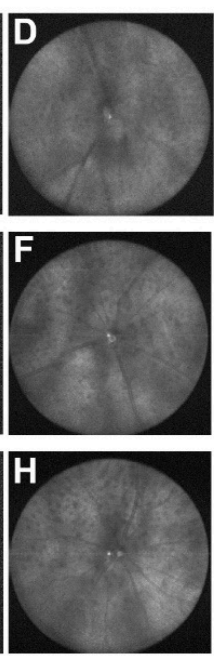

K

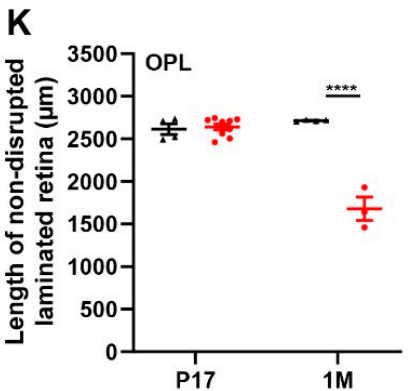

\section{Crb1 mutant}
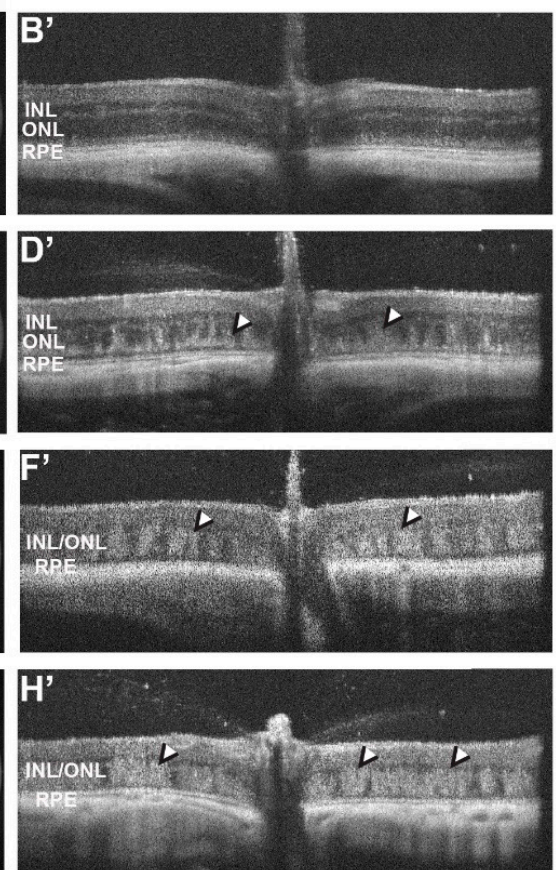

L

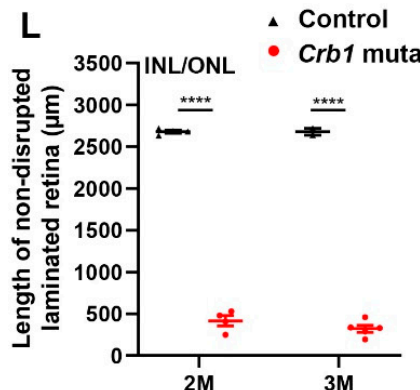

Figure 3. In vivo imaging of $\mathrm{Crb1}$ mutant rats show increased retinal degeneration over time. Volume intensity projection (VIP) of the P17 and 1, 2 and 3 months of age control (A,C,E,G) and Crb1 mutant (B,D,F,H) rat retinas; and SD-OCT B-scans of the P17 and 1, 2 and 3 months of age control $\left(\mathbf{A}^{\prime}, \mathbf{C}^{\prime}, \mathbf{E}^{\prime}, \mathbf{G}^{\prime}\right)$ and $C r b 1$ mutant rat retinas $\left(\mathbf{B}^{\prime}, \mathbf{D}^{\prime}, \mathbf{F}^{\prime}, \mathbf{H}^{\prime}\right)$. Quantifications of total retinal thickness (I), length of non-disrupted laminated retina at the OLM in P17 and 1-month-old control and Crb1 mutant rats (J), at the OPL (K), and in 2 and 3 months of age rats at the INL/ONL (L). Arrowheads indicate regions of retinal disorganization. Number of animals used for P17 (control $n=6, C r b 1$ mutant = 9), $1 \mathrm{M}$ (control $n=4, C r b 1$ mutant $n=9$ ), $2 \mathrm{M}$ (control $n=4, \mathrm{Crb1}$ mutant $n=4)$ and 3M (control $n=2, \mathrm{Crb1}$ mutant $n=5$ ). Values are presented as the mean \pm SEM. ${ }^{* * *} p<0.001,{ }^{* * * *} p<0.0001$. INL $=$ inner nuclear layer; ONL $=$ outer nuclear layer; OPL $=$ outer plexiform layer; $\mathrm{RPE}=$ retinal pigment epithelium. 
Quantification of the total retinal thickness at all ages revealed a significant decrease in the Crb1 mutant compared to control rat retinas (Figure 3I). In addition, the length of disrupted and normal laminated retina was determined in both the control and Crb1 mutant rat retina at all ages analyzed. At P17, both control and Crb1 mutant rat retinas mainly showed a normal retinal lamination with a limited number of disruptions at the OLM and the outer plexiform layer (OPL) (Figure 3J,K). At one month of age, a significant increase in the length of degenerated retina were observed, indicated by OLM breaks (Figure 3J) or disruptions at the OPL in the Crb1 mutant rats (Figure 3K). Finally, at two and three months of age, the length of areas of INL and ONL disruptions increased in the $\mathrm{Crb1}$ mutant rats (Figure 3L). Altogether, these data demonstrate the early-onset severe degenerative phenotype observed in $\mathrm{Crb1}$ mutant rats over time.

\subsection{Ultra-Structural Localization of CRB1 and CRB2 Proteins in the Control and Crb1 Mutant Rat Retina}

CRB1 and CRB2 localization was studied by immunohistochemistry in the P5 control and Crb1 mutant rat retinas. Canonical CRB1 was found at the OLM in control rats, while the CRB1 ${ }^{\mathrm{INDEL}}$ protein was below the detection level in the $\mathrm{Crb1}$ mutant rat retina (Figure 4A,B). CRB2 was detected at the OLM in both control and Crb1 mutant rats (Figure 4C,D). Interestingly, the CRB2 staining is more diffuse in the Crb1 mutant in comparison to control rats (Figure $4 \mathrm{C}, \mathrm{D}$ ). In addition, the onset of retinal function loss in the $\mathrm{Crb1}$ mutant rats is remarkably faster than in mice lacking CRB1 $\left(\mathrm{Crb} 1^{\mathrm{KO}}\right)$, mice carrying a missense CRB1 mutation $\left(\mathrm{Crb}^{\mathrm{KO} / \mathrm{C} 249 \mathrm{~W}}\right)$ or the naturally occurring $\mathrm{Crb1} \mathrm{rd} 8$ mouse $[3,5,17,18]$. Previously, we found that CRB1 or CRB2 proteins localized differently in adult human retina, fetal human retina, human iPSC-derived retinal organoids and non-human-primate retina compared to mouse retina $[8,19]$. For that reason, we performed immuno-electron microscopy to determine the localization of canonical CRB1 and CRB2 in the control compared to Crb1 mutant rat retinas. In control rats, CRB1 was abundantly present at the subapical region in MGCs but not photoreceptors at both P17 and 3 months of age (Figures $4 \mathrm{E}, \mathrm{G}$ and $5 \mathrm{~A}$ ), while the $\mathrm{CRB} 1^{\mathrm{INDEL}}$ variant protein in $\mathrm{Crb1}$ mutant rats was only sporadically and at very low levels detected at the subapical region in MGCs (Figure 4F,H). For CRB2, a different pattern was observed: CRB2 is present at the subapical region of the MGCs and photoreceptors in both control and Crb1 mutant retinas (Figures 4I-L and 5B). The cellular localizations of CRB1 and CRB2 are therefore similar in the mouse and rat. 
CRB1

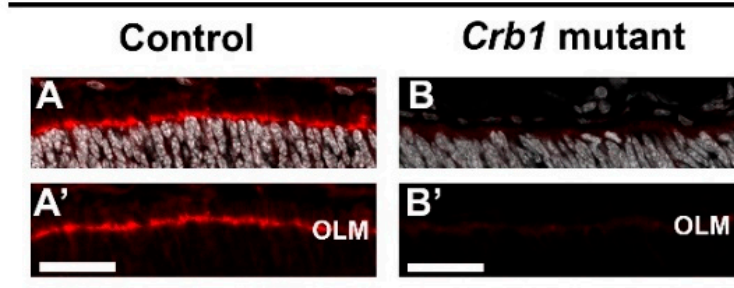

P17
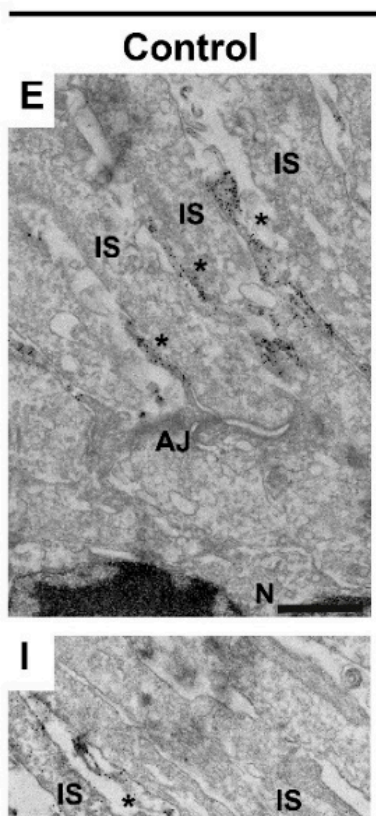

CRB2

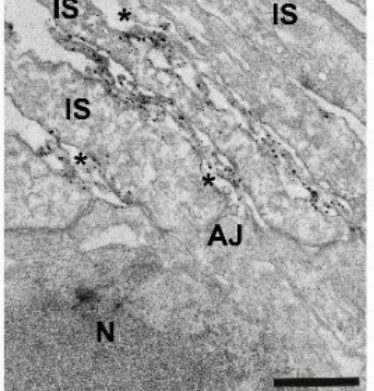

is
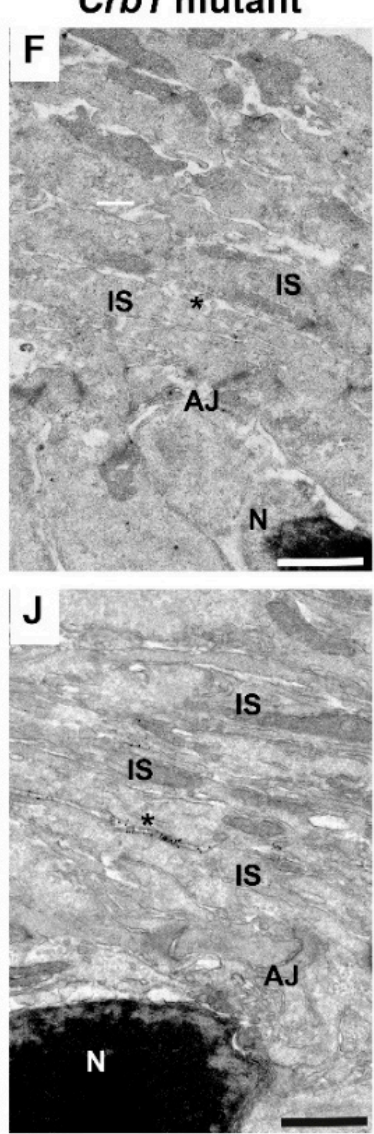

CRB2

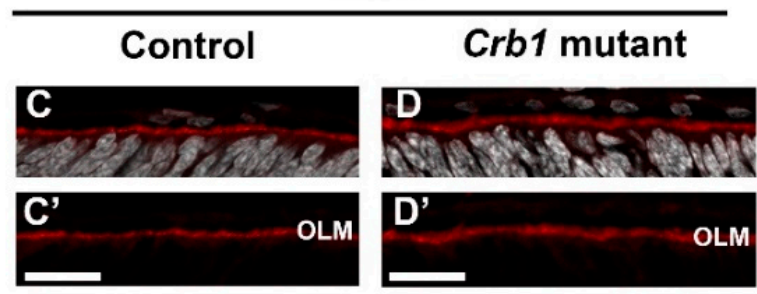

$3 \mathrm{M}$
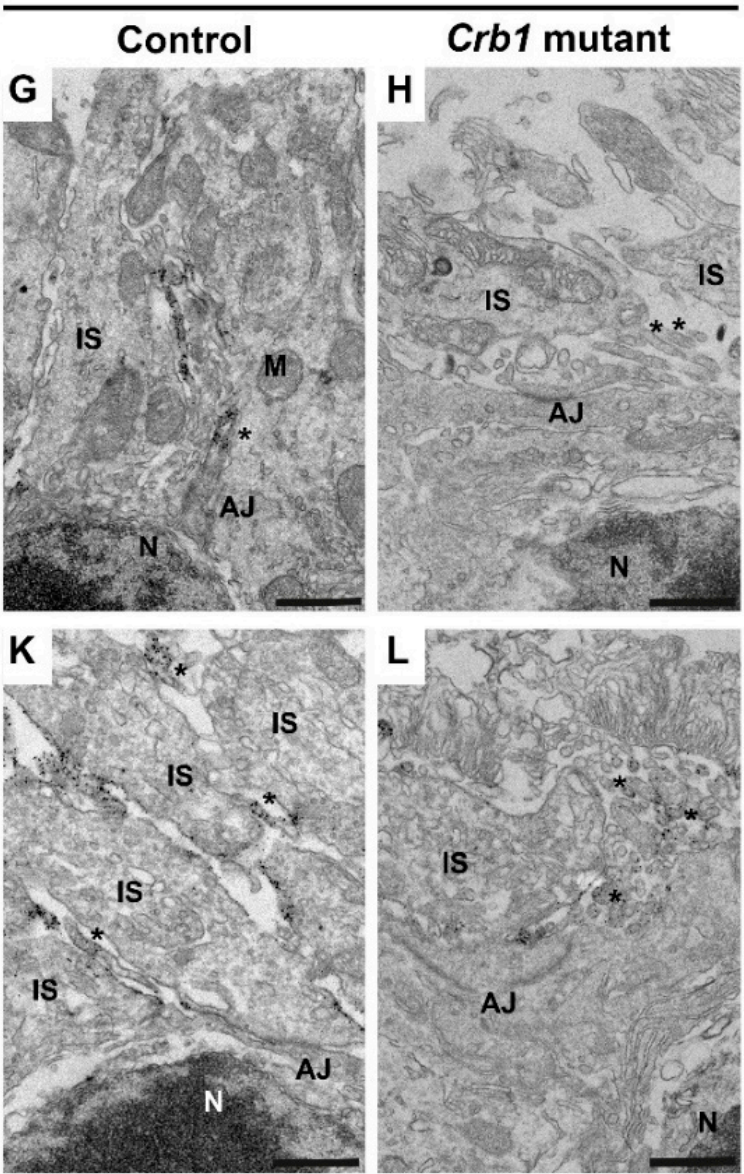

Figure 4. CRB1 is present at the subapical region (SAR) of MGCs in control rats while CRB2 is present at the SAR of both photoreceptors and MGC in control and Crb1 mutant rats. (A-D) Immunohistochemical analysis of CRB1 (A,B) and CRB2 (C,D) in P5 control and Crb1 mutant rats at the OLM. (E-L) Subcellular localization of CRB1 and CRB2 in control and Crb1 mutant rats using immuno-electron microscopy. CRB1 is present at the SAR of MGC in both P17 (E) and 3M control retina (G), while the mutant $\mathrm{CRB1}{ }^{\mathrm{INDEL}}$ variant protein in Crb1 mutant rats is sporadically detectable in P17 (F) and $3 \mathrm{M}(\mathbf{H})$. F and $\mathrm{H}$ show representative images lacking detectable label. CRB2 is present at the SAR of MGCs as well as photoreceptors in both control and Crb1 mutant rat retinas at both ages (I-L). $n=2$ animals used per time point. AJ = adherens junctions; IS = inner segments; INL = inner nuclear layer; $\mathrm{M}=$ mitochondria; $\mathrm{N}=$ nucleus; $\mathrm{OLM}=$ outer limiting membrane; $\mathrm{ONL}=$ outer nuclear layer; ${ }^{*}=$ Müller glial villi. Scale bars $(\mathbf{A}-\mathbf{D})=20 \mu \mathrm{m} ;(\mathrm{E}-\mathrm{L})=1 \mu \mathrm{m}$. 


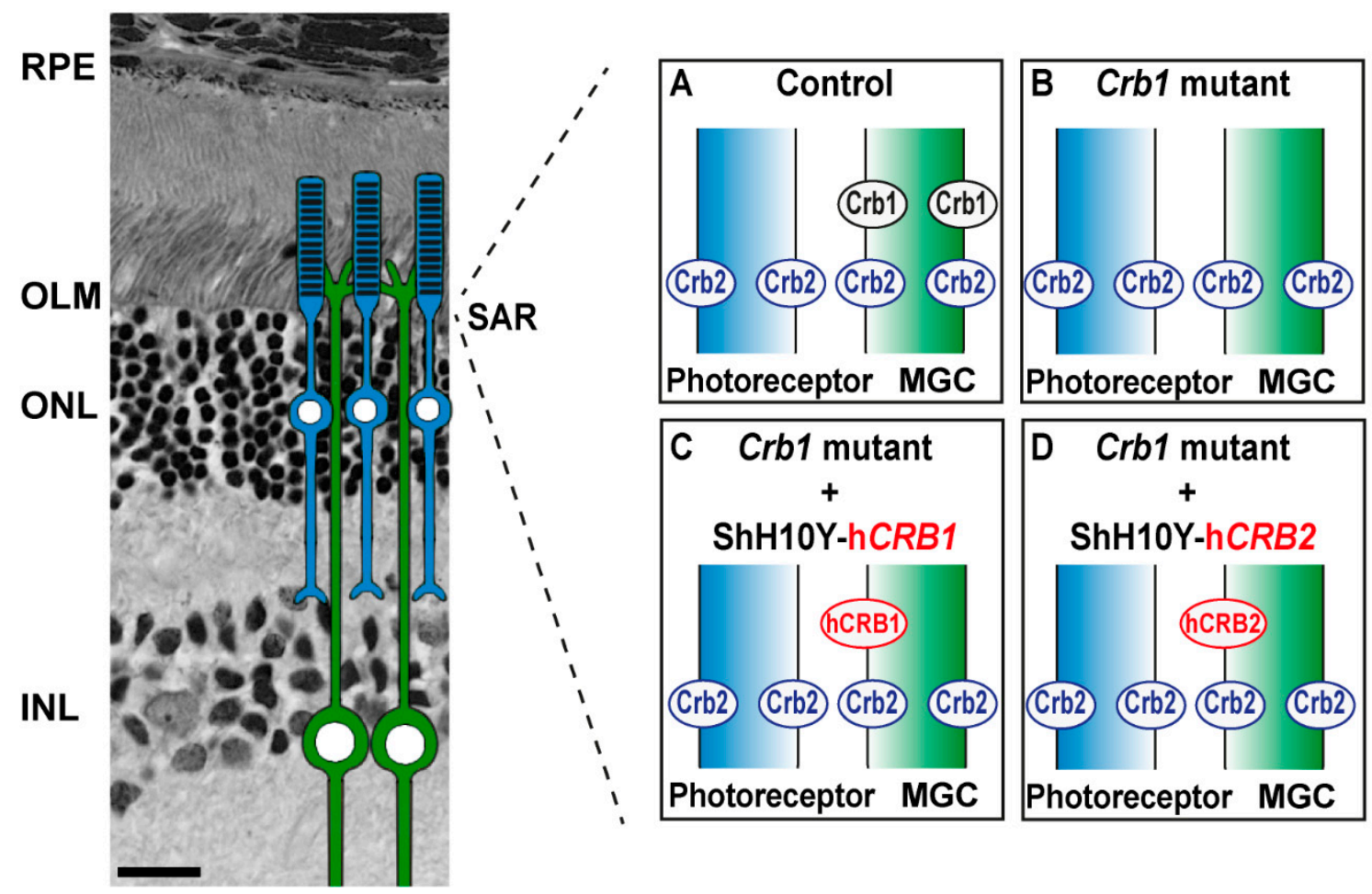

Figure 5. Graphical representation of subcellular localization of Crb1 and Crb2 proteins in Brown Norway rat retina and gene therapy approach using ShH10Y-hCRB1 and ShH10Y-hCRB2. Graphical representation of the subcellular localization of $\mathrm{Cb} 1$ and $\mathrm{Crb} 2$ in control (A) and Crb1 mutant (B) rat retinas. Graphical representation using AAV-mediated gene therapy vectors targeting MGCs using ShH10Y-hCRB1 (C) and ShH10Y-hCRB2 (D). Scale bar: $20 \mu \mathrm{m}$. RPE = retinal pigment epithelium; OLM = outer limiting membrane; ONL = outer nuclear layer; INL = inner nuclear layer; $\mathrm{SAR}=$ subapical region; MGC = Müller glial cell.

\subsection{Adeno-Associated Viral Vector (AAV) Tropism in Young Brown Norway Rat Retina}

To define the tropism of different AAV capsids, $1 \mu \mathrm{L}$ of $1 \times 10^{13} \mathrm{gc} / \mathrm{mL}$ AAV2-CMVGFP expression vectors packaged into three different AAV serotypes (AAV2/5.CMV.GFP, AAV2/9.CMV.GFP and AAV2/ShH10 ${ }^{445 F}$.CMV.GFP, called from now on AAV5, AAV9 and $\mathrm{ShH} 10 \mathrm{Y}$, respectively) were intravitreally or subretinally injected before retinal degeneration in P5 and P8 in control and Crb1 mutant rat retinas. One month after injection, eyes were collected and analyzed using immunohistochemistry. Differences in retinal tropism were observed using the distinct application methods in new-born control and Crb1 mutant rats; a summary of the tropism in the different serotypes, routes and time points of delivery is shown in Table 1. Interestingly, no difference in tropism was observed between control and Crb1 mutant rats, nor the time point of the injection (P5 or P8; Table 1). 
Table 1. Summary of the retinal tropism of three different adeno-associated viral vector (AAV) serotypes, namely, AAV5, AAV9 and ShH10Y, injected via two different routes of delivery at P5 or P8 in control and Crb1 mutant rat retina. For each serotype and route of delivery, a dose of $\sim 1 \times 10^{13} \mathrm{gc} / \mathrm{mL}$ was injected in a volume of $1 \mu \mathrm{L}$. Quantified data are presented as follows: no GFP positive (-); 1 GFP positive (+/ -); 2-5 GFP positive (+); 6-10 GFP positive (++); 11-15 GFP positive $(+++)$; and $\geq 16$ GFP positive cells $(++++)$ per $100 \mu \mathrm{m}$. Number of animals analyzed: AAV5 control P5 SR $n=6$, P5 IV $n=2$, P8 SR $n=2$, P8 IV $n=2$, AAV5 Crb1 mutant P5 SR $n=4$, P5 IV $n=3$, P8 SR $n=2$, P8 IV $n=5$; AAV9 control P5 SR $n=3$, P5 IV $n=2$, P8 SR $n=3$, P8 IV $n=3$, AAV9 Crb1 mutant P5 SR $n=4$, P5 IV $n=3$, P8 SR $n=2$, P8 IV $n=3$; ShH10Y control P5 SR $n=2, \mathrm{P} 5 \mathrm{IV} n=6$, P8 SR $n=2$, P8 IV $n=5$, ShH10Y Crb1 mutant P5 SR $n=3$, P5 IV $n=4, \mathrm{P} 8 \mathrm{SR} n=2$, P8 IV $n=3$. DOI = date of injection; TOI = type of injection; GCL = ganglion cell layer; RNFL = retinal nerve fiber layer; INL = inner nuclear layer; $\mathrm{ONL}$ = outer nuclear layer; $\mathrm{RPE}=$ retinal pigment epithelium; $\mathrm{SR}=$ subretinal injection; IV = intravitreal injection.

\begin{tabular}{|c|c|c|c|c|c|c|c|}
\hline AAV & DOI & TOI & GCL/RNFL & INL & ONL & RPE & \\
\hline \multirow{9}{*}{ AAV5 } & P5 & SR & - & - & ++ & + & Control \\
\hline & P5 & SR & - & - & + & + & Crb1 mutant \\
\hline & P5 & IV & - & $+/-$ & $+/-$ & - & Control \\
\hline & P5 & IV & - & - & + & - & Crb1 mutant \\
\hline & P8 & SR & - & $+/-$ & +++ & + & Control \\
\hline & P8 & SR & - & - & +++ & + & Crb1 mutant \\
\hline & P8 & IV & - & - & + & - & Control \\
\hline & P8 & IV & - & + & $+/-$ & - & Crb1 mutant \\
\hline & P5 & SR & - & - & ++ & + & Control \\
\hline \multirow{7}{*}{ AAV9 } & P5 & SR & - & - & +++ & + & Crb1 mutant \\
\hline & P5 & IV & - & ++ & $+/-$ & - & Control \\
\hline & P5 & IV & $+/-$ & + & - & - & Crb1 mutant \\
\hline & P8 & SR & - & - & +++ & + & Control \\
\hline & P8 & SR & - & - & +++ & + & Crb1 mutant \\
\hline & P8 & IV & - & $+/-$ & - & - & Control \\
\hline & P8 & IV & - & $+/-$ & - & - & Crb1 mutant \\
\hline \multirow{8}{*}{ ShH10Y } & P5 & SR & - & + & + & + & Control \\
\hline & P5 & SR & - & + & + & + & Crb1 mutant \\
\hline & P5 & IV & - & +++ & $+/-$ & - & Control \\
\hline & P5 & IV & - & ++ & + & - & Crb1 mutant \\
\hline & P8 & SR & - & + & + & + & Control \\
\hline & P8 & SR & - & + & + & + & Crb1 mutant \\
\hline & P8 & IV & - & ++ & - & - & Control \\
\hline & P8 & IV & - & ++ & $+/-$ & - & Crb1 mutant \\
\hline
\end{tabular}

Subretinal injection of AAV5 and AAV9 at both P5 and P8 resulted in predominantly transduced RPE and photoreceptors, whereas intravitreal injection of both serotypes showed transduction of photoreceptors and, to a lesser extent, transduction of INL cells (Figure 6A-P). Furthermore, subretinal injection of ShH10Y demonstrated infection of RPE cells, photoreceptors and MGCs (Figure 6Q,R,U,V), while intravitreal injection of ShH10Y resulted mainly in transduction of MGCs and other cell types in the INL (Figure 6S,T,W,X). Co-staining with GS confirmed the transduction of mainly MGCs with all ShH10Y injections performed (Figure S2). 

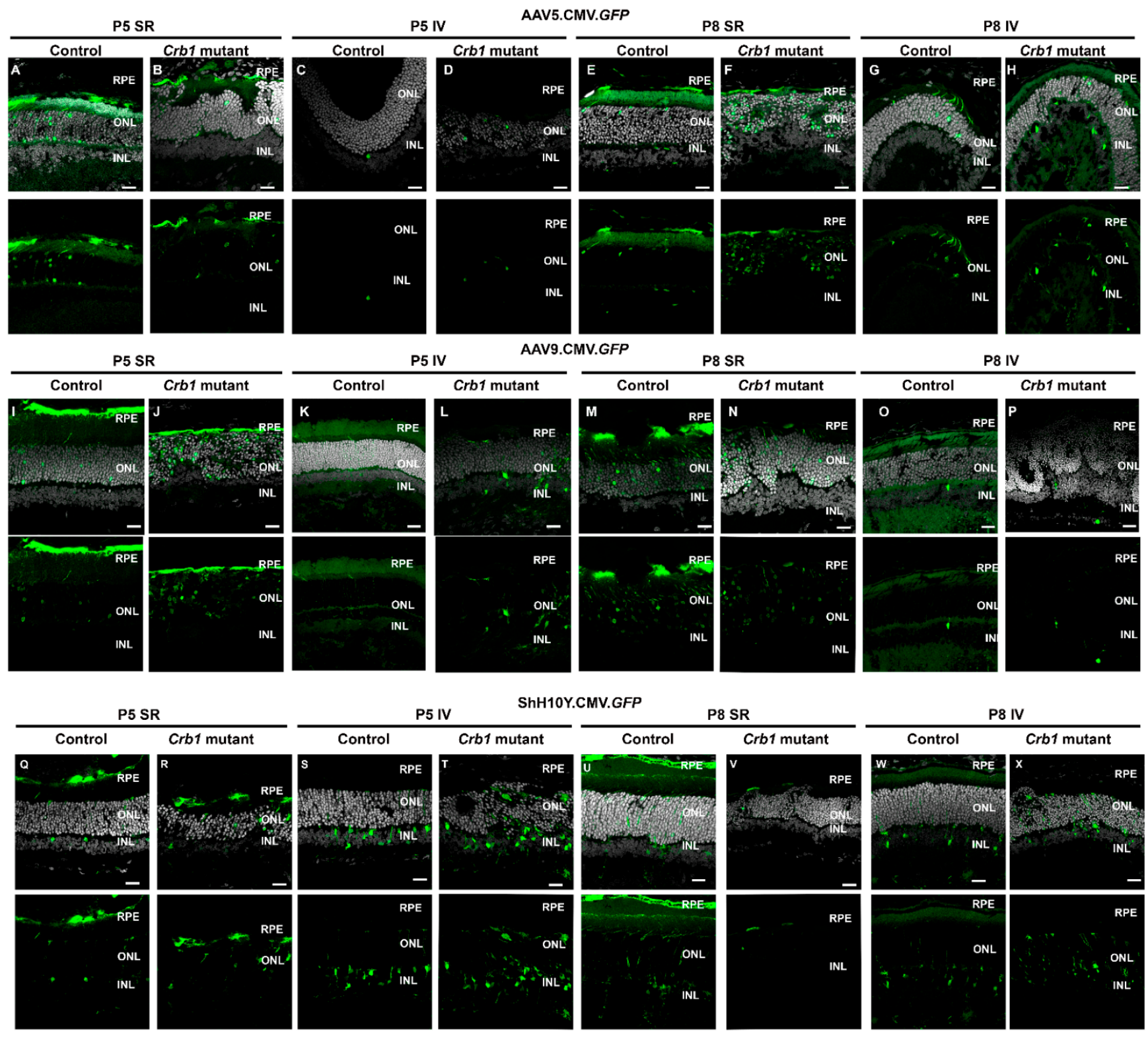

Figure 6. Retinal tropisms of AAV5, AAV9 and ShH0Y in new-born Crb1 mutant and control rat retinas. P5 or P8 control and Crb1 mutant rats were injected subretinally (SR) or intravitreally (IV) with either AAV5-, AAV9- or ShH10Y.CMV.GFP and analyzed at 1 month of age. Tropism studies show that SR injection of AAV5 and AAV9 mainly infect RPE and photoreceptors at P5 (A,B,I,J); similar results were obtained when injected at P8 (E,F,M,N). IV injection at P5 of AAV5 and AAV9 show mainly infection of sporadic photoreceptors and some INL cells $(\mathbf{C}, \mathbf{D}, \mathbf{K}, \mathbf{L})$; similar results were obtained when injected at P8 (G,H,O,P). SR injection of ShH10Y at both P5 and P8 in control and Crb1 mutant retinas results in infection of RPE, photoreceptors and INL cells $(\mathbf{Q}, \mathbf{R}, \mathbf{U}, \mathbf{V})$; analysis of IV injection of ShH10Y at both P5 and P8 show transduction of INL cells $(\mathbf{S}, \mathbf{T}, \mathbf{W}, \mathbf{X})$. For each serotype and route of delivery, a dose of $\sim 1 \times 10^{13} \mathrm{gc} / \mathrm{mL}$ was injected in a volume of $1 \mu \mathrm{L}$. Scale bar: $20 \mu \mathrm{m}$. Number of animals analyzed: AAV5 control P5 SR $n=6$, P5 IV $n=2$, P8 SR $n=2$, P8 IV $n=2$, AAV5 Crb1 mutant P5 SR $n=4, \mathrm{P} 5 \mathrm{IV} n=3, \mathrm{P} 8 \mathrm{SR} n=2$, P8 IV $n=5$; AAV9 control P5 SR $n=3, \mathrm{P} 5 \mathrm{IV} n=2, \mathrm{P} 8 \mathrm{SR} n=3, \mathrm{P} 8 \mathrm{IV} n=3$, AAV9 Crb1 mutant P5 SR $n=4$, P5 IV $n=3$, P8 SR $n=2$, P8 IV $n=3$; ShH10Y control P5 SR $n=2$, P5 IV $n=6$, P8 SR $n=2$, P8 IV $n=5$, ShH10Y Crb1 mutant P5 SR $n=3$, P5 IV $n=4$, P8 SR $n=2, \operatorname{P8} \operatorname{IV} n=3$.

\subsection{Intravitreal Delivery of ShH10Y-hCRB1 or ShH10Y-hCRB2 at P5 Does Not Increase the} Retinal Function in Crb1 Mutant Rats

Based on the subcellular localization of CRB1 and the tropism of the different AAV capsids, we determined that intravitreal injection of $\mathrm{ShH10Y}$ is the most suitable viral vector 
to infect MGCs and explore gene therapy possibilities in Crb1 mutant rats (Figure 5C,D). In addition, tropism studies in both control and $\mathrm{Cr} b 1$ mutant rats show that larger stretches of the retina are transduced when intravitreally injected at P5 with ShH10Y (Figure S3A,B). For that reason, the Crb1 mutant rats were injected at P5, with ShH10Y either with hCRB1 (ShH10Y-hCRB1) or hCRB2 (ShH10Y-hCRB2) and the other eye was injected with PBS (mock control). Immunohistochemical analysis of 3-month-old Crb1 mutant rats injected at P3 with ShH10Y-hCRB1 show expression of hCRB1 above the OLM (Figure S3C). In addition, rats injected with ShH10Y-hCRB2 reveal a remaining CRB2 localization at the OLM similar to the uninjected condition (Figure S3D). The visual and retinal function were measured by OKT in 3-month-old, and ERG at 2- and 3-month-old Crb1 mutant rats. No significant differences were observed in visual function, as measured by the OKT spatial frequency, between untreated, PBS-, ShH10Y-hCRB1- or ShH10Y-hCRB2-treated eyes at 3 months of age (Figure 7A). In addition, no significant difference in retinal function as measured by ERG was observed between ShH10Y-hCRB1-treated and PBS-injected eyes (Figure 7B,C). However, an increase in OKT contrast sensitivity at $0.092 \mathrm{c} / \mathrm{d}$ spatial frequency was observed in 3-month-old Crb1 mutant rats injected with ShH10Y-hCRB2 compared to PBS-injected rats (Figure 7A). In addition, an increased ERG response at high dark-adapted intensities (1.5 and 1.9) on the a-wave amplitude for rod photoreceptor transmission was observed in Crb1 mutant rats treated with ShH10Y-hCRB2 (Figure 7C).

When the retinal function within individual rats was analyzed in 2- and 3-months-old Crb1 mutant rats, no significant differences were observed between ShH10Y-hCRB1 and their PBS-injected eyes for both the scotopic a- and b-wave (Figure 7D and Figure S3E). In addition, no differences were observed when treated with ShH10Y-hCRB2 (Figure 7E and Figure S3F). Interestingly, we observed high variations on the scotopic ERG responses between individual animals measured injected with PBS, ShH10Y-hCRB1 or ShH10Y-hCRB2 (a-wave range 10-100 $\mu \mathrm{V}$; b-wave range $50-300 \mu \mathrm{V}$; Figure 7D,E); similar variations were observed when the response of the right and left eye of untreated $\mathrm{Crb1}$ mutant rats were compared (a-wave range 10-100 $\mu \mathrm{V}$; b-wave range 100-300 $\mu \mathrm{V}$; Figure 7F), indicating a variability in the scotopic ERG response between different $\mathrm{Crb1}$ mutant litters. Because of the absence of an enhanced retinal function after gene therapy delivery, we hypothesize that the injection with our viral vectors at P5 does not allow timely expression of the hCRB1 or hCRB2 transgenes before the onset of loss of visual or retinal function in the Crb1 mutant rats. 
A
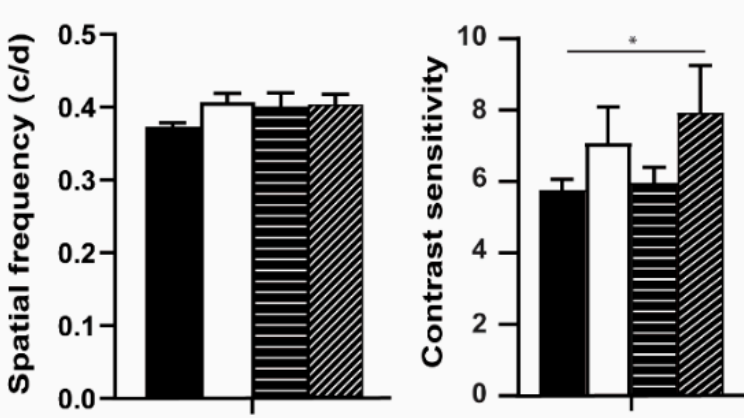

\author{
Uninjected \\ $\square$ PBS injected \\ = ShH10Y-hCRB1 \\ WII \\ ShH10Y-hCRB2
}

B
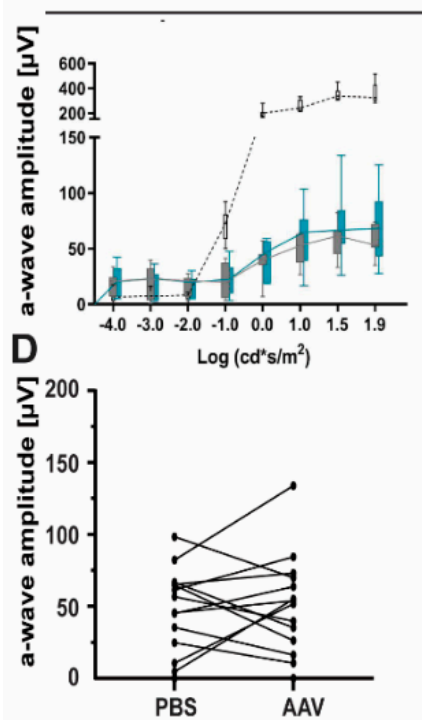

F

\section{Uninjected Crb1 mutant}
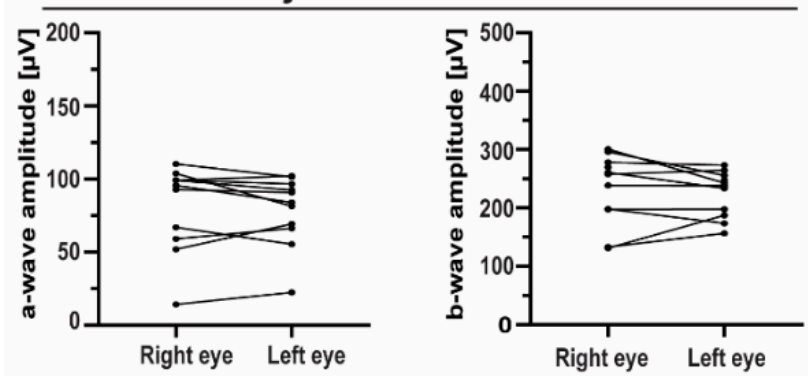

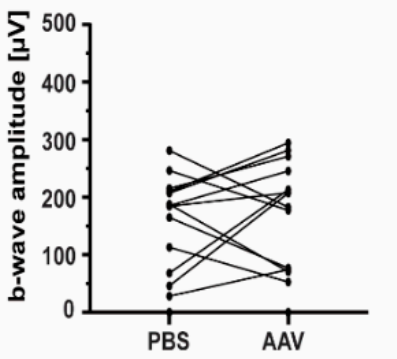

C

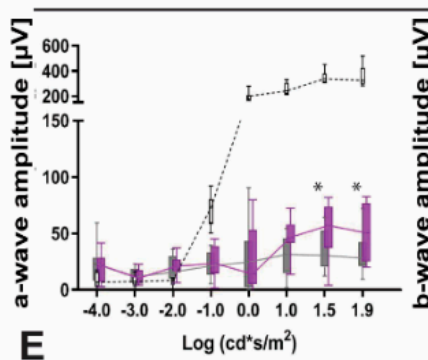

ShH10Y-hCRB2

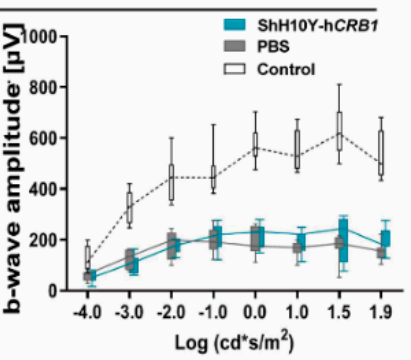

E

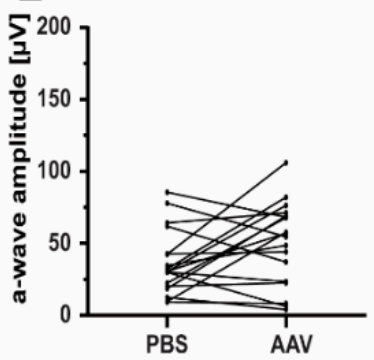

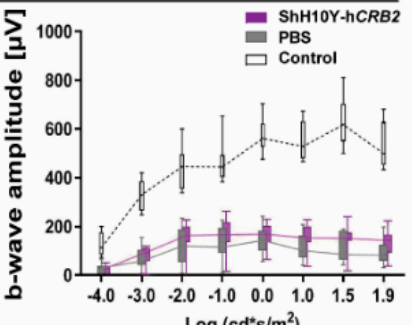

$\log \left(\mathrm{cd}^{*} \mathrm{~s} / \mathrm{m}^{2}\right)$

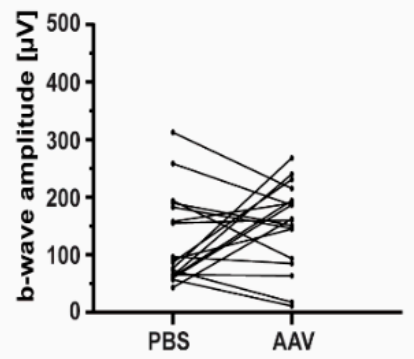

Figure 7. Retinal function in 3M rats after intravitreal injection at P5 of ShH10Y-hCRB1 or ShH10Y-hCRB2 is not restored. (A) OKT spatial frequency and 0.092 contrast sensitivity of 3M rats injected with PBS, ShH10Y-hCRB1, or ShH10Y-hCRB2. $(\mathbf{B}, \mathbf{C})$ Quantification of all scotopic a- and b-wave ERG at 3M of the group of rats injected with ShH10Y-hCRB1 (B) or ShH10Y-hCRB2 (C) with uninjected eye and a wild-type as the controls. (D,E) 1.5 stimulus intensity ERG at 3M comparison of individual rats injected with either ShH10Y-hCRB1 (D) or ShH10Y-hCRB2 (E) compared with PBS-injected eyes. (F) 1.5 stimulus intensity ERG at 3M comparison of the left and right eye of non-injected Crb1 mutant rats. Boxes indicate the 25 and $75 \%$ quantile range and whiskers indicate the 5 and $95 \%$ quantiles, and the intersection of line and error bar indicates the median of the data (box-and-whisker plot). ${ }^{*} p<0.05$. Number of animals used: ShH10Y-hCRB1 $n=7,3 \mathrm{M}$ OKT and $n=15$ 3M ERG; and for ShH10Y-hCRB2 injected $n=15$ 3M OKT, and $n=18$ 3M ERG. Mean \pm SEM.

\title{
3. Discussion
}

In this study, we demonstrate (1) progressive retinal degeneration in Crb1 mutant rats, causing loss of visual and retinal function; (2) ultrastructural localization of CRB1 at the subapical region of MGCs, and CRB2 at the subapical region of MGCs and photoreceptors in control rats; (3) a decrease in CRB1 ${ }^{\text {INDEL }}$ at the subapical region of MGCs in Crb1 mutant 
rats; (4) tropism by subretinal and intravitreal application of AAV5, AAV9 and ShH10Y in control and Crb1 mutant rats; and (5) AAV-hCRB gene therapy at P5 for MGCs of Crb1 mutant rats does not result in a functional rescue.

The Crb1 mutant rats exhibit a significant decreased ERG response at 1 month of age in comparison with age-matched control rats, which is in accordance with previously published results comparing the Crb1 mutant to BN-Harlan rats [10]. We further expand the characterization of the Crb1 mutant rat strain by showing a reduced OKT spatial frequency response in 3-month-old Crb1 mutant rats compared to age-matched control rats. Interestingly, although the control rats show a reduced ERG response from 1 month of age onwards, the OKT spatial frequency decline is minor. This discrepancy has been described before, where similar spatial frequencies only declined months after significant photoreceptor and ERG response loss [20].

Here, we observed the first signs of retinal degeneration from P10 in the Crb1 mutant rat retina, including photoreceptor nuclei protrusions in the photoreceptor segment layers and OLM breaks at foci throughout the entire retina. These disruptions are ultimately resulting in large regions with a complete disorganized ONL and INL lamination in adult $\mathrm{Cr} b 1$ mutant rats. Interestingly, the phenotype at P10 in the Crb1 mutant rats is comparable to previously described Crb1 mouse models [3,17], but the retinal phenotype in these rats at 1 month of age is much more severe. In addition, in $\mathrm{Crb} 1$ mouse models, the retinal degeneration is limited to the inferior quadrant $[3,17]$, whereas in the Crb1 mutant rats it is presented throughout the entire retina. These discrepancies could be because of (1) different genetic backgrounds, including species differences; (2) different types of mutations affecting different CRB isoforms [21,22], thereby expressing a distinct CRB1 $1^{\mathrm{INDEL}}$ protein in the Crb1 mutant rats; or (3) the total expression levels of CRB2 might be significantly lower in new-born rats compared to new-born mice. Decreased levels of CRB2 or dysregulation of other CRB-interacting proteins could result in less stabilization of the adherens junction complex at the OLM, resulting in a more severe phenotype. In addition, transcriptomic analysis identified several dysregulated pathways in $C r b 1$ mutant rats, such as TGF- $\beta$ signaling, matrix metalloproteinases, type II interferon signaling, MAPK cascade, inflammatory pathways, regulation of actin cytoskeleton and many more [10]. More research is required to define which factors play a major role in the retinal degeneration in these $\mathrm{Cr} b 1$ mutant rats.

SD-OCT imaging allows to follow the retinal degeneration over time in $\mathrm{Crb1}$ mutant compared to age-matched control rat retinas. Previous research using SD-OCT with $\mathrm{Crb1}$ rd8 or $C r b 1^{\text {lowMGC }}$ mouse models show typical ocular lesions, such as pseudo-rosette formation $[13,23,24]$. Here, we observed a relatively healthy retinal lamination in P17 Crb1 mutant rat retina with some sporadic disruptions at the OLM. At one month of age, the retinal lamination is disrupted throughout the retina, indicated by hyperreflective regions at the ONL. These hyperreflective disruptions are thought to begin in the OLM because of adhesion abnormalities between the photoreceptor and MGCs. Over time, in 2- and 3-month-old rats, an increased degeneration with larger hyperreflective regions, indicating a potential lack of retinal lamination, is observed. Similar results were obtained using histological sections. Like human CRB1-RD patients [24], hyperreflective lesions of various sizes were observed in the $\mathrm{Cr} b 1$ mutant rat starting at 1 month of age. The hyperreflective ONL and INL in the Crb1 mutant rats made automated as well as manual segmentation of the layers challenging, particularly at 2 and 3 months of age. Therefore, we quantified the length of the observed disruptions and compared it to the length of the retinal view, hereby distinguishing between the length of disrupted and normal laminated retinas. Quantification of the data showed a significant increase in OLM breaks and disruptions at the OPL in the Crb1 mutant compared to age-matched control rats at 1,2 and 3 months of age. Several researchers have shown that quantification of retinal layer size by SD-OCT, compared with histological sections, contain less variations, presumably due to artefacts from the post-mortem processing [25]. Altogether, these data are showing the importance of the SD-OCT imaging over time in Crb1 mutant rats. 
Immunohistochemical analysis reveal the localization of CRB2 at the subapical region (SAR) in control and Crb1 mutant rats, while canonical CRB1 is only detected at the SAR of control rats. The CRB1 antibody used detects the carboxyl terminus of the CRB1 protein, and our data show that the Crb1 mutant rats lack the full length CRB1 at the OLM. In control rats, subcellular localization of CRB1 and CRB2 by immuno-electron microscopy revealed the presence of CRB1 at the SAR adjacent to the adherens junctions in MGCs, and of CRB2 at the SAR in both MGCs and photoreceptors. These data correspond well to the localization previously found in mouse studies [26]. In postmortem human cadaver retinas, however, CRB1 localized at the subapical region in both MGCs and photoreceptor. In these studies, CRB2 localized at the SAR in MGCs, with CRB2 localized at vesicles in the photoreceptor inner segments $[12,27]$. Interestingly, recent data revealed the subcellular localization of both CRB1 and CRB2 at the SAR of MGCs as well as photoreceptors in human iPSCderived retinal organoids, second trimester human fetal retina and non-human-primate retinas $[8,19]$. The cellular localization of CRB1 and CRB2 in Brown Norway rats are therefore only similar to mice.

The $\mathrm{Crb1}$ gene locus is complex with various $\mathrm{Crb1}$ splice forms and gene products from alternate promoters, such as Crb1-B [17,21,28]. Full-length canonical Crb1 is encoded on 12 exons with the highly conserved CRB1 carboxyl terminus encoded on exon-12, whereas the $C r b 1-B$ lacks the conserved carboxyl terminus since encoded on $5^{\prime}$-alternate exon- $5 \mathrm{a}$, 6-11 alternate- $3^{\prime}$ [21]. The in-frame insertion-deletion mutation in exon 6 of the $C r b 1$ mutant rat also affects the $C r b 1-B$ gene, and it is to be tested whether the mutation specifically in $C r b 1-B$ contributes to the observed early-onset severe retinal phenotype. Interestingly, $\mathrm{Cr} b 1$ rd8 mice have an out-of-frame base-pair deletion in exon- 9 that causes an alternate- $3^{\prime}$ and encodes, therefore, for two truncated transcripts (upstream promoter driving expression of $C r b 1$ exons 1-6 indel, 7-9 alternate- $3^{\prime}$; and a promoter in intron-5 driving expression of Crb1 exons 5a-6indel, 7-9 alternate-3') [17]. However, the mutation affecting these two gene products from $\mathrm{Crb1} \mathrm{rd} 8$ do not result in a severe retinal degeneration as observed in the Crb1 mutant rats, suggesting the existence of other modifying factors that play a role in the severity of the retinal phenotype. Reduced levels of CRB2 in MGCs and/or photoreceptors or retinal progenitors in mice lacking CRB1 result in a significant more severe retinitis pigmentosa or Leber congenital amaurosis retinal phenotype $[8,27]$. Altogether, these data suggest the existence of other modifying factors that play a role in the severity of the retinal phenotype observed in $\mathrm{Crb1}$ mutant rats. It remains of interest to test whether low levels of CRB2 contribute to the early-onset severe retinal phenotype observed in new-born Crb1 mutant rats, whereas high levels of CRB2 might suppress the onset of retinal phenotype in Crb1 mice.

For AAV-mediated gene therapy purposes, in new-born $C r b 1$ mutant rats, we determined the retinal tropism of three different AAV serotypes (AAV5, AAV9, ShH10Y) upon different routes of AAV delivery. The AAV capsids were injected at different time points (P5 or P8), either subretinal or intravitreal, in both control and Crb1 mutant rats. Even though retinal injection efficiency can vary between animals, the transduced layers of cells with a specific capsid and type of injection was similar between animals injected at the two different time points. With subretinal delivery, the serotypes AAV5 and AAV9 successfully transduced the RPE and photoreceptors in new-born control and Crb1 mutant rats, while subretinal delivery of ShH10Y was transducing RPE, photoreceptors, MGCs and other cell types in the INL. Subretinal delivery of AAV9 at P5 and P8 in both control and Crb1 mutant rats results in a similar transduction pattern as observed by others after subretinal injection in two-month old Sprague-Dawley rats [29]. In addition, AAV5 tropism at P5 and P8 in control and Crb1 mutant rats is similar as described in 6- to 8-week-old C57/BL6 wild-type mice [30]. When AAV.CMV.GFP vectors were injected intravitreally in new-born control and $\mathrm{Crb1}$ mutant rats, serotypes AAV5 and AAV9 showed a relatively poor transduction efficiency of both photoreceptors and other cells in the INL. Other researchers showed a poor transduction of cells in the INL as well upon intravitreal delivery of AAV2, AAV6 and AAV8 in two-month-old Sprague-Dawley rats [29]. However, we observed efficient 
transduction of mainly MGCs in the INL after intravitreal injection in new-born control and Crb1 mutant rats with ShH10Y, which is consistent with previously published data obtained in the adult rat [31].

Transduction efficiency in the degenerated retina might differ from that in the healthy control retina [30,32]. However, we did not observe a difference in cellular tropism nor in the transduction efficiency between control and $\mathrm{Crb1}$ mutant rats with all serotypes and the expression vector AAV.CMV.GFP tested. Moreover, no differences in tropism were observed when all AAV serotypes were injected either at P5, before the onset of retinal degeneration or at $\mathrm{P} 8$, closer to the first signs of degeneration. This might be explained by the correctly laminated retina in the new-born $\mathrm{Crb1}$ mutant rat retina, and it is well possible that AAV tropism might differ when injected at later time points where the degeneration is more advanced. In summary, we highlight the important differences of AAV capsids and types of delivery that could be considered with future gene therapy approaches.

Finally, we explored the possibility of AAV-mediated hCRB gene therapy in Crb1 mutant new-born rats. Based on the tropism results, we used intravitreal injection of ShH10YhCRB1 and ShH10Y-hCRB2 at P5 in Crb1 mutant rats for our gene therapy experiments. The efficacy of these ShH10Y-hCRB gene therapy vectors was shown previously $[12,13]$. When individual untreated $\mathrm{Crb1}$ mutant rat ERG responses were analyzed at different time points, no differences were detected between the left and right eyes within the same animal. This suggests that both eyes show similar rates of loss of retinal function.

Lack of an improved visual function after AAV-CRB application could be caused by the time lag for $\mathrm{AAV}$-mediated gene therapy; it might take several days to weeks before the hCRB transgene in Crb1 mutant rats is expressed at full level in the target cells, in this case the target cells being the MGCs. In our tropism studies, we observed upon intravitreal injection of $1 \mu \mathrm{L} 1 \times 10^{13} \mathrm{gc} / \mathrm{mL}$ ShH10Y-hCRB at P5 a good but potentially incomplete transduction of $\mathrm{Crb1}$ mutant rat MGCs. In other studies, an increased b-wave was observed when 10-day-old Royal College of Surgeons rats were subretinally injected with a total of $8 \mu \mathrm{L}$ containing $4 \times 10^{8}$ particles $(4 \mu \mathrm{L}$ superior hemisphere and $4 \mu \mathrm{L}$ in the inferior hemisphere) of AAV2.CMV.Mertk to target defective RPE [33]. Whereas, in our $C r b 1$ mutant rat studies, $1 \mu \mathrm{L}$ of a dose of $1 \times 10^{13} \mathrm{gc} / \mathrm{mL}$ was injected intravitreally at P5, which might be a too low dose of ShH10Y-hCRB1 or ShH10Y-hCRB2 to slow down the retinal degeneration observed in the $C r b 1$ mutant rats. In addition, $1 \mu \mathrm{L}$ of our gene therapy vector might not spread well throughout the entire retina. Improving the surgical application technique to enlarge the transduced area of the retina could be considered [34] Moreover, we observed a wide variability in ERG response between different Crb1 mutant rat litters, likely because of the Brown Norway genetic background. Backcrossing these rats into a more defined genetic background might decrease the observed variability in retinal phenotype. In $\mathrm{Crb} 1^{\mathrm{rd} 8 / \mathrm{rd} 8}$ mice on a C57BL/6 genetic background, considerable variation in retinal phenotype was observed as well [35]. In addition, the injection efficiency and thereby the number of AAV viral particles taken up by the retinal cells might vary between animals. Moreover, there might be differences in intracellular release of AAV-DNA from capsids inside the targeted cells. Finally, proof-of-concept studies showed functional and structural preservation in a Crb1 mouse model by using AAV2/9.CMV.hCRB2 [12] or AA2/ShH10Y.CMV.hCRB2 [13]. However, the retinal phenotypes in these mice were less severe than the one observed at 1 and 3 months of age $\mathrm{Crb1}$ mutant rats. In previous studies, we showed that human CRB2 can compensate for the loss of endogenous Crb proteins in the mouse retina $[12,13]$. Here, we tested human CRB1 or CRB2 in gene therapy studies to compensate for the loss of functional $\mathrm{Crb} 1$ protein in the rat retina. We showed expression of hCRB1 in Crb1 mutant rats after intravitreally injected ShH10Y-hCRB1 (Figure S3C), but we did not observe an enhanced retinal function after gene therapy delivery. In silico analysis show that both CRB1 and CRB2 are highly conserved proteins between rat and human (Figure S4). Despite the high sequence similarity, proteins may have distinct species-specific properties or activity that need to be taken into account when designing gene therapy studies. Here, we did not test the application of rat $\mathrm{Crb1}$ or $\mathrm{Crb2}$ gene therapy 
vectors to the rat retina due to ethical issues, as the expected outcome of such experiments on a large group of rats is presumed to be negative. Therefore, we cannot exclude the possibility that rat $\mathrm{Crb} 1$ or $\mathrm{Crb} 2$ proteins could alleviate the loss of endogenous rat Crb1 in models with slower onset of retinal degeneration. All these differences could explain the lack of functional rescue observed here; thus, future experiments could focus on the development of novel gene therapy vectors that allow immediate early-onset expression of transgenes at P5 or low molecular weight drug therapy in new-born rats, or in utero gene therapy approaches.

In conclusion, we further characterized the early-onset morphological phenotype and the retinal function of $C r b 1$ mutant rats in comparison to age-matched control rats. In addition, we show that the ultrastructural localization of endogenous CRB1 and CRB2 in the rat retina is similar as observed in the mouse retina. In addition, as little is known about AAV tropism administered in new-born Brown Norway rats, we showed differences in cellular tropism when three different AAV capsids were injected in both Crb1 mutant and control rats. Finally, no timely rescue of the retinal phenotype was observed using retinal function and visual acuity, suggesting the need for earlier onset of expression of recombinant hCRB proteins in Müller glial cells to rescue the severe retinal phenotype in Crb1 mutant rats.

\section{Materials and Methods}

\subsection{Animals}

Procedures concerning animals were performed in accordance with the EU Directive 2010/63/EU for animal experiments and with permission of the Dutch Central Authority for Scientific Procedures on Animals (CCD), permit number 1160020172924, approved 18, January, 2018. The animals were maintained on a $12 \mathrm{~h}$ day-night cycle and were supplied ad libitum with food and water. Brown Norway rats from Janvier Labs with a spontaneous mutation in the Crb1 gene were used in this study [10]; the Crb1 mutant rat breeding was set up within the LUMC animal facility. Age-matched control Brown Norway rats lacking the mutation in the $\mathrm{Cr} b 1$ gene from Charles River Laboratories were used as controls. Animals were killed by carbon dioxide inhalation.

\subsection{DNA Isolation and Genetic Analysis}

The presence of spontaneous in-frame insertion-deletion (indel) in exon 6 of the Crb1 gene was validated using DNA extraction from rat's tails by proteinase K digestion overnight at $55^{\circ} \mathrm{C}$ in lysis buffer $(100 \mathrm{mM}$ Tris- $\mathrm{HCl}, \mathrm{pH} 8.5,5 \mathrm{mM}$ EDTA, 0.2\% SDS, and $200 \mathrm{mM} \mathrm{NaCl}$ ). Biopsies were centrifuged for $15 \mathrm{~min}$, the supernatant was transferred and mixed vigorously with isopropanol, followed by a 10 min centrifuge at $14,000 \mathrm{rpm}$ and removal of the supernatant. Then, the pellet was washed twice with $80 \%$ ethanol, air dried for $10 \mathrm{~min}$ at $55^{\circ} \mathrm{C}$ and subsequently resuspended in $200 \mu \mathrm{L} \mathrm{10 \%} \mathrm{TE}(10 \mathrm{mM}$ Tris- $\mathrm{HCl}, \mathrm{pH} 8.0$, $1 \mathrm{mM}$ EDTA). Finally, DNase was inactivated for $15 \mathrm{~min}$ at $65^{\circ} \mathrm{C}$. For genotyping, PCR with primers targeting the location of the INDEL in the $C r b 1$ gene was performed and was subsequently sequenced using Sanger Sequencing, FW: 5'-TTCAGACTGTTCAGCCAAATGC-3', REV: 5'-TGTCCCCATTGGTAAGCCACC-3'.

\subsection{Electroretinography (ERG)}

Dark- and light-adapted ERGs were performed under dim red light using an Espion E2 (Diagnosys, LLC, MA). ERGs were performed on 1, 2 and 3-month-old control and Crb1 mutant rats. Rats were anesthetized using $100 \mathrm{mg} / \mathrm{kg}$ ketamine and $10 \mathrm{mg} / \mathrm{kg}$ xylazine intraperitoneally and the pupils were diluted using tropicamide drops $(5 \mathrm{mg} / \mathrm{mL})$. Rats were placed on a heating pad, and reference and ground platinum electrodes were placed subcutaneously and in the base of the tail, respectively. ERGs were recorded from both eyes using gold wire electrodes. Hypromellose eye drops $(3 \mathrm{mg} / \mathrm{mL}$, Teva) were given between recordings to prevent eyes from drying. Single (scotopic and photopic ERG) or brief train (Flicker ERG) white (6500k) flashes were used. The band-pass filter frequencies 
were 0.3 and $300 \mathrm{~Hz}$. Scotopic recordings were obtained from dark-adapted animals at the following light intensities: $-4,-3,-2,-1,0,1,1.5$ and $1.9 \log \mathrm{cd} \mathrm{s} / \mathrm{m} 2$ [36]. Flicker recordings were obtained under a fixed light intensity of $0.5 \log \mathrm{cd} \mathrm{s} / \mathrm{m} 2$ with varying frequency $(0.5,1,2,3,5,7,10,12,15,18,20$ and $30 \mathrm{~Hz})[37,38]$. Photopic recordings were performed following $10 \mathrm{~min}$ light adaptation on a background light intensity of $30 \mathrm{~cd}^{*} \mathrm{~m} 2$ and the light intensity series used was: $-2,-1,0,1,1.5,1.9 \log \mathrm{cd}^{*} \mathrm{~s} / \mathrm{m} 2$ [36]. The numbers of rats used per time point are indicated under the designated figure. For the gene therapy studies, responses of the treated eye, either right or left eye, were compared with the other eye, mock injected (PBS), at each time point analyzed.

\subsection{Optokinetic Tracking Reflex (OKT)}

Spatial frequency and contrast sensitivity thresholds were measured using an optomotor system (Cerebral Mechanics, Lethbridge, AB, Canada). Two and three month(s)-old rats were placed on a small platform in the center of four computer monitors that formed a virtual drum with a rotating vertical sine wave grating $\left(12^{\circ} / \mathrm{s}(\mathrm{d} / \mathrm{s})\right)$, as described previously [14]. Head movements in the same direction as the rotating gratings were considered as positive responses and no response was considered as negative response. Spatial frequency thresholds were determined with an increasing staircase paradigm, starting at 0.042 cycles/deg (c/d) with $100 \%$ contrast. Contrast sensitivity thresholds were measured across three spatial frequencies $(0.092 \mathrm{c} / \mathrm{d})$. The reciprocal of the contrast sensitivity threshold was used as the contrast sensitivity value at each spatial frequency.

\subsection{Morphological Analysis}

Eyes were collected at a range of time points from P5 to 3-month-old control and Crb1 mutant rats ( $n=2-4$ /age/group). For morphological analysis, eyes were enucleated and fixed with $4 \%$ paraformaldehyde in phosphate buffered saline (PBS) for $20 \mathrm{~min}$ at room temperature. After fixation, the eyes were dehydrated for $30 \mathrm{~min}$ in 30, 50, 70, 90 and 99\% ethanol. Subsequently, the eyes were embedded in Technovit 7100 (Kulzer, Wehrheim, Germany) and sectioned $(3 \mu \mathrm{m})$ as previously described [39]. Slides were dried, counterstained with $0.5 \%$ toluidine blue and mounted under coverslips using Entellan (Merk, Darmstadt, Germany). Eye sections were scanned using a Pannoramic 250 digital slide scanner (3DHISTECH Ltd., Budapest, Hungary) and images were processed with CaseViewer 2.1 (3DHISTECH Ltd., Budapest, Hungary).

\subsection{Immunohistochemical Analysis}

Eyes were collected at a range of time points from P5 to 3-month-old control and Crb1 mutant rats $(n=2-4 /$ age/group). For immunohistochemical analysis, eyes were enucleated and fixed with $4 \%$ paraformaldehyde in PBS for $20 \mathrm{~min}$ at room temperature. Then, the eyes were cryo-protected with $15 \%$ and $30 \%$ sucrose in PBS, embedded in Tissue-Tek O.C.T Compound (Sakura, Finetek), and used for cryosectioning. Cryosections of $8 \mu \mathrm{m}$ were made with a Leica CM1900 cryostat (Leica Microsystems).

Sections for immunohistochemistry were blocked for $1 \mathrm{~h}$ at RT in $10 \%$ normal goat serum, $0.4 \%$ Triton $\mathrm{X}-100$ and $1 \%$ bovine serum albumin (BSA) in PBS. The primary antibodies were diluted in $0.3 \%$ normal goat serum, $0.4 \%$ Triton X-100 and $1 \%$ BSA in PBS and incubated in a moist chamber overnight at $4{ }^{\circ} \mathrm{C}$. After rinsing in PBS, the sections were incubated for $1 \mathrm{~h}$ at RT with the fluorescent-labelled secondary antibodies goat antimouse, goat anti-rabbit or goat anti-chicken IgGs conjugated to Alexa 488, Alexa 555 (1:1000; Abcam) or Cy3 (1:500), which were diluted in 0.1\% goat serum in PBS. Nuclei were counterstained with DAPI and mounted in a Vectashield Hardset mounting medium (H1500 or H1800, Vector Laboratories, Burlingame, USA). Sections were imaged on a Leica TCS SP8 confocal microscope. Confocal images were processed with Leica Application Suite X (v3.7.0.20979).

The following primary antibodies were used: P120-catenin (1:250; BD Biosciences Cat\# 610134), CRB1 AK2 (1:200; homemade), CRB2 SK11 (1:200; [3]), glutamine synthetase (GS) 
(1:250; BD Biosciences Cat\# 610518), PALS1 (1:200; homemade), recoverin (1:500; Millipore Cat\# AB5585) and rhodopsin (1:500; Millipore Cat\# MAB5356), SOX9 (1:250; Millipore Cat\# AB5535).

\subsection{Spectral Domain Optical Coherence Tomography (SD-OCT)}

P17, 1, 2 and 3 month control and $\mathrm{Crb1}$ mutant rats were anesthetized using $60 \mathrm{mg} / \mathrm{kg}$ ketamine and $60 \mathrm{mg} / \mathrm{kg}$ xylazine $(50 \mathrm{mg} / \mathrm{kg}$ ketamine and $5 \mathrm{mg} / \mathrm{kg}$ xylazine for P17 rats) intraperitoneally and the pupils were diluted using tropicamide drops $(5 \mathrm{mg} / \mathrm{mL})$. Anesthetized rats were placed in front of the SD-OCT imaging device (EnvisuTM R2210 VHR, Leica, USA). Eyes were kept moisturized with Vidisic Carbogel and Systane ultraeyedrops during the whole procedure. Image acquisitions were performed using the following parameters: rectangular scans of $3.2 \mathrm{~mm}$ by $3.2 \mathrm{~mm}$, A-scans/B-scans: 1000, B-scans: 100, Frames/B-scan: 6 (for high resolution B-scans); and A-scans/B-scans: 400, B-scans: 400, Frames/B-scan: 4 (also known as the isotropic scan for an enface projection image). Thickness of retinal layers were manually measured using Bioptigen InVivoVue Reader and Diver software in the individual layers at $0.3,0.6,0.9$ and $1.3 \mathrm{~mm}$ both sides from the center of the optic nerve head in the nasal-temporal direction. In addition, the length of disrupted and healthy retinal lamination was measured using Fiji ImageJ software, where the frame with the optic nerve head and $800 \mu \mathrm{m}$ before and after the optic nerve head in nasal-temporal directions were used for quantification. Values of the three different frames in the left and right eye were averaged and plotted in the figure together; so, one value per animal.

\subsection{Immuno-Electron Microscopy}

Immuno-electron microscopy was performed as previously described [40]. In brief, $40 \mu \mathrm{m}$ sections were incubated with a primary antibody for $48 \mathrm{~h}$, it was then incubated with a secondary peroxidase anti-peroxidase for $4 \mathrm{~h}$. After that, sections were developed in a 2,2-diaminobenzidine solution for 4-8 $\mathrm{min}$, and then the gold-substitute-silver-peroxidase method was applied. Sections were then prepared for electron microscopy and overlapping images were collected using a One View Camera (Gatan) as previously described [41].

\subsection{Delivery of the $A A V$}

For tropism experiments, five days old rats were anesthetized using hypothermia and eight days old rats were anesthetized using an intraperitoneally injected with $35 \mathrm{mg} / \mathrm{kg}$ ketamine and $35 \mathrm{mg} / \mathrm{kg}$ xylazine. Eyelids were opened and eyes were popped out using surgical tools, the pupils were dilated with $1 \%$ tropicamide drops $(5 \mathrm{mg} / \mathrm{mL})$ and kept moist with Hypromellose drops. Under visualization with an operating microscope intravitreal and subretinal injections were performed in control and $\mathrm{Cr} b 1$ mutant rats. We used AAV2/5, AAV2/9 or AAV2/ShH10 ${ }^{\mathrm{Y} 445 \mathrm{~F}}$, with the full-length CMV promotor, GFP and bovine growth hormone polyadenylation sequence for our tropism study. For each serotype and route of delivery, a dose of $\sim 1 \times 10^{13} \mathrm{gc} / \mathrm{mL}$ was injected in a volume of $1 \mu \mathrm{L}$ using a 33-gauge blunt-tipped Hamilton syringe (Hamilton Company, Reno, NV, USA). Eyes were closed and protected with "Hansaplast liquid protection" and treated with ointment containing chloramphenicol to prevent infections.

For the gene therapy experiments, similar procedures were followed as described above. Here, one eye was treated with an AAV vector and the other eye was mockinjected (PBS); this was randomized in the left and right eye. We used intravitreal injection of ShH10Y capsids to package the AAV2 inverted terminal repeats, the full length or minimal CMV promoter (CMV, or CMVmin), hCRB1 or hCRB2 cDNAs and synthetic poly-adenylation sequence for our gene therapy experiments. The AAVs were spiked with 1/10 dose ShH10Y, with the full-length CMV promotor, GFP cDNA and bovine growth hormone polyadenylation sequence. We carefully examined the quality of our AAV-hCRB vector preparations by qPCR and Western blots; we recently showed before that these batches of $A A V$ vectors worked efficiently in $h C R B$ gene therapy studies [13]. 


\subsection{Tropism Quantification}

Immunohistochemical slides for tropism studies were imaged on a Leica TCS SP8 confocal microscope, and sections were scanned for AAV-GFP-positive areas to re-confirm the subretinal or intravitreal injection and define the tropism. For each condition, $4-8$ confocal images spanning the area of transduction of each eye was acquired $(n \geq 2$ independently injected eyes for each condition). Each individual confocal image was acquired at 40X magnification. The number of eGFP-positive cells within each layer were manually counted. The total number of eGFP-positive cells per eye were divided by transduction diameter to determine the number of positive cells per $100 \mu \mathrm{m}$. Then eyes were averaged and coded as - = 0 GFP+ cells; + / - ; 1 GFP+ cells, $+; 2-5$ GFP + cells, $++; 6-10$ GFP + cells, +++; 11-15 GFP+ cells, ++++ ; and $>16$ GFP+ cells.

\subsection{Statistical Analysis}

We performed statistical analysis for group comparisons: comparing the untreated Crb1 mutant and age-matched control rats by ERG using a two-way ANOVA; comparing all AAV-treated eyes with the other untreated or PBS-injected eyes using a two-way ANOVA (at 2 and 3 months of age); comparing the AAV-treated eye with the other untreated or PBS-injected eyes using a paired t-test; and, finally, statistical analysis on SD-OCT quantifications using a two-way ANOVA. These statistical analyses were performed using GraphPad Prism version 8 (GraphPad Software). All values are expressed as the mean \pm SEM if not otherwise indicated. Statistically significant values: ${ }^{*} p<0.05 ;{ }^{* *} p<0.01$; *** $p<0.001 ;{ }^{* * * *} p<0.0001$.

\section{Patents}

The LUMC is the holder of patent application PCT/NL2014/050549, which describes the potential clinical use of CRB2; J.W. is listed as an inventor on this patent, and J.W. is an employee of the LUMC.

Supplementary Materials: The following are available online at https:/ /www.mdpi.com/article/10 $.3390 /$ ijms $22073563 / \mathrm{s} 1$. Figure S1. Retinal function further decreases in 3 and 5 months of age Crb1 mutant compared to control rats. Quantitative analysis of the scotopic a-wave (A) and b-wave (B), the photopic b-wave (C), the b-wave/a-wave amplitude and b/a-ratios (b/a) (D), and the flicker amplitude response (E) in 3 and 5 months of age $\mathrm{Crb1}$ mutant and control rats. Boxes indicate the 25 and $75 \%$ quantile range and whiskers indicate the 5 and $95 \%$ quantiles, and the intersection of line and error bar indicates the median of the data (box-and-whisker plot). Number of animals used for 3 months: control $=8, \mathrm{Crb1}$ mutant $=6$, and $5 \mathrm{M}$ : control $=6, \mathrm{Crb1}$ mutant $=6$. Mean $\pm \mathrm{SEM} .{ }^{*} p<0.05$; ${ }^{* *} p<0.01 ; * * *<0.001 ; * * * * p<0.0001$. Figure S2. Predominantly MGC transduction upon subretinal or intravitreal delivery of ShH10Y in both control and $\mathrm{Crb1}$ mutant rat retina. ShH10Y transduction at $\mathrm{P} 5$ subretinal $(\mathrm{A}, \mathrm{B})$, or intravitreal $(\mathrm{C}, \mathrm{D})$, and $\mathrm{P} 8$ subretinal $(\mathrm{E}, \mathrm{F})$, or intravitreal $(\mathrm{G}, \mathrm{H})$ in one month control $(\mathrm{A}, \mathrm{C}, \mathrm{E}, \mathrm{G})$ or $\mathrm{Crb1}$ mutant $(\mathrm{B}, \mathrm{D}, \mathrm{F}, \mathrm{H})$ rats retina. Co-stained with glutamine synthetase (GS; red), co-localization of GFP and GS indicated with arrowheads. At least $n=2$ eyes used per time point. $\mathrm{INL}=$ inner nuclear layer, $\mathrm{ONL}=$ outer nuclear layer. $\mathrm{SR}=$ subretinal injection, $\mathrm{IV}=$ intravitreal injection. Scale bar: $20 \mu \mathrm{m}$. Figure S3. Gene therapy of ShH10Y-hCRB1 or ShH10Y-hCRB2 at P5 does not improve the severe retinal phenotype measured by ERG analysis at $2 \mathrm{M}$ of age. Overview of control (A) and Crb1 mutant (B) rats intravitreally injected at P5 with ShH10Y-GFP, indicating the area of transduction. Immunohistochemical analysis of 3 months old $\mathrm{Crb1}$ mutant rats intravitreally injected at P3 with ShH10Y-hCRB1 (C) or with ShH10Y-hCRB2 (D) revealing the expression of hCRB1 and hCRB2. And 1.5 stimulus intensity ERG comparison of 2M individual rats injected with either ShH10Y-hCRB1 (E) or ShH10Y-hCRB2 (F) at P5 compared with PBS injected eyes. Number of animals used: P5 injection with ShH10Y-hCRB1 $n=11$; ShH10Y-hCRB2 $n=9$. INL = inner nuclear layer, OLM $=$ outer limiting membrane, $\mathrm{ONL}=$ outer nuclear layer. Scale bar: (A,B) $200 \mu \mathrm{m},(\mathrm{C}, \mathrm{D}) 20 \mu \mathrm{m}$. Figure S4. Highly conserved CRB1 and CRB2 protein sequence between human and Brown Norway rats. Protein sequence alignment of human CRB1 with rat Crb1 (A) and human CRB2 with rat Crb2 (B). Protein sequence alignment reveals $75 \%$ identical matches and $84 \%$ conservative substitutions for human CRB1 compared with rat Crb1. And for human CRB2 compared with rat $\mathrm{Crb2}$ there are $77 \%$ 
identical matches and $82 \%$ conservative substitutions shown. Upper and bold sequence is human, lower sequence is rat. Sequence alignment data from Uniprot and BLAST.

Author Contributions: Conceptualization, N.B., C.H.A. and J.W.; formal analysis, N.B., C.H.A., A.A.M., C.A.A., C.R.J. and J.W.; funding acquisition, J.W.; investigation, N.B., C.H.A. and J.W.; methodology, N.B., C.H.A., A.A.M., C.A.A., T.M.B. and P.M.J.Q.; project administration, J.W.; resources, T.M.B., P.M.J.Q. and R.M.V.; supervision, J.W.; validation, N.B., C.H.A., A.A.M., C.A.A., C.R.J. and J.W.; visualization, N.B.; writing-original draft, N.B.; writing-review and editing, N.B., C.H.A., A.A.M., C.A.A., T.M.B., P.M.J.Q., A.J.K., C.R.J. and J.W. All authors have read and agreed to the published version of the manuscript.

Funding: This research was funded by Foundation Fighting Blindness (TA-GT-0715-0665-LUMC, to JW), the Netherlands Organization for Health Research and Development (ZonMw grant 43200004, to JW), Bontius Stichting (Bontius 23122019 to JW) and the Dutch blindness funds (Uitzicht 2013-13 to JW, Uitzicht 2018-6 to CHA and JW): Rotterdamse Stichting Blindenbelangen, MaculaFonds, Stichting Blindenhulp, Landelijke Stichting voor Blinden en Slechtzienden, Algemene Nederlandse Vereniging ter Voorkoming van Blindheid, Stichting Blinden-Penning.

Institutional Review Board Statement: The study was conducted according to the guidelines of the EU Directive 2010/63/EU for animal experiments and with permission of the Dutch Central Authority for Scientific Procedures on Animals (CCD), permit number 1160020172924.

Informed Consent Statement: Not applicable.

Data Availability Statement: The data that support the findings of this study are available from the corresponding author upon reasonable request.

Acknowledgments: The authors thank Chelsey Linnenbank, Arend Boogaard, and Nynke van der Haar for technical assistance and all members of the Wijnholds lab for advice on the manuscript.

Conflicts of Interest: The LUMC is the holder of patent application PCT/NL2014/050549, which describes the potential clinical use of CRB2; J.W. is listed as an inventor on this patent, and J.W. is an employee of the LUMC.

\section{References}

1. Den Hollander, A.I.; Ten Brink, J.B.; De Kok, Y.J.M.; Van Soest, S.; Van Den Born, L.I.; Van Driel, M.A.; Van De Pol, D.J.R.; Payne, A.M.; Bhattacharya, S.S.; Kellner, U.; et al. Mutations in a human homologue of Drosophila crumbs cause retinitis pigmentosa (RP12). Nat. Genet. 1999, 23, 217-221. [CrossRef]

2. Roh, M.H.; Makarova, O.; Liu, C.J.; Shin, K.; Lee, S.; Laurinec, S.; Goyal, M.; Wiggins, R.; Margolis, B. The Maguk protein, Pals1, functions as an adapter, linking mammalian homologues of crumbs and discs lost. J. Cell Biol. 2002, 157, 161-172. [CrossRef]

3. van de Pavert, S.A.; Kantardzhieva, A.; Malysheva, A.; Meuleman, J.; Versteeg, I.; Levelt, C.; Klooster, J.; Geiger, S.; Seeliger, M.W.; Rashbass, P.; et al. Crumbs homologue 1 is required for maintenance of photoreceptor cell polarization and adhesion during light exposure. J. Cell Sci. 2004, 117, 4169-4177. [CrossRef]

4. Margolis, B. The Crumbs3 polarity protein. Cold Spring Harb. Perspect. Biol. 2018, 10, 1-9. [CrossRef]

5. $\quad$ van de Pavert, S.A.; Meuleman, J.; Malysheva, A.; Aartsen, W.M.; Versteeg, I.; Tonagel, F.; Kamphuis, W.; McCabe, C.J.; Seeliger, M.W.; Wijnholds, J. A single amino acid substitution (Cys249Trp) in Crb1 causes retinal degeneration and deregulates expression of pituitary tumor transforming gene Pttg1. J. Neurosci. 2007, 27, 564-573. [CrossRef] [PubMed]

6. Pellissier, L.P.; Alves, C.H.; Quinn, P.M.; Vos, R.M.; Tanimoto, N.; Lundvig, D.M.S.; Dudok, J.J.; Hooibrink, B.; Richard, F.; Beck, S.C.; et al. Targeted ablation of $\mathrm{Crb} 1$ and $\mathrm{Crb} 2$ in retinal progenitor cells mimics leber congenital amaurosis. PLoS Genet. 2013, 9. [CrossRef] [PubMed]

7. Alves, C.H.; Pellissier, L.P.; Vos, R.M.; Garrido, M.G.; Sothilingam, V.; Seide, C.; Beck, S.C.; Klooster, J.; Furukawa, T.; Flannery, J.G.; et al. Targeted ablation of Crb2 in photoreceptor cells induces retinitis pigmentosa. Hum. Mol. Genet. 2014, 23, 3384-3401. [CrossRef] [PubMed]

8. Quinn, P.M.; Mulder, A.A.; Henrique Alves, C.; Desrosiers, M.; de Vries, S.I.; Klooster, J.; Dalkara, D.; Koster, A.J.; Jost, C.R.; Wijnholds, J. Loss of CRB2 in Müller glial cells modifies a CRB1-associated retinitis pigmentosa phenotype into a Leber congenital amaurosis phenotype. Hum. Mol. Genet. 2019, 28, 105-123. [CrossRef] [PubMed]

9. Quinn, P.M.; Alves, C.H.; Klooster, J.; Wijnholds, J. CRB2 in immature photoreceptors determines the superior-inferior symmetry of the developing retina to maintain retinal structure and function. Hum. Mol. Genet. 2018, 27, 3137-3153. [CrossRef] [PubMed]

10. Zhao, M.; Andrieu-Soler, C.; Kowalczuk, L.; Paz Cortés, M.; Berdugo, M.; Dernigoghossian, M.; Halili, F.; Jeanny, J.-C.; Goldenberg, B.; Savoldelli, M.; et al. A new CRB1 rat mutation links müller glial cells to retinal telangiectasia. J. Neurosci. 2015, 35, 6093-6106. [CrossRef] [PubMed] 
11. Wang, D.; Tai, P.W.L.; Gao, G. Adeno-associated virus vector as a platform for gene therapy delivery. Nat. Rev. Drug Discov. 2019, 18, 358-378. [CrossRef]

12. Pellissier, L.P.; Quinn, P.M.; Alves, C.H.; Vos, R.M.; Klooster, J.; Flannery, J.G.; Wijnholds, J. Gene therapy into photoreceptors and Müller glial cells restores retinal structure and function in CRB1 retinitis pigmentosa mouse models. Hum. Mol. Genet. 2015, 24, 1-15. [CrossRef] [PubMed]

13. Buck, T.M.; Vos, R.M.; Alves, C.H.; Wijnholds, J. AAV-CRB2 protects against vision loss in an inducible CRB1 retinitis pigmentosa mouse mode. Mol. Ther. Methods Clin. Dev. 2021, 20, 423-441. [CrossRef]

14. Prusky, G.T.; Alam, N.M.; Beekman, S.; Douglas, R.M. Rapid quantification of adult and developing mouse spatial vision using a virtual optomotor system. Investig. Ophthalmol. Vis. Sci. 2004, 45, 4611. [CrossRef] [PubMed]

15. Douglas, R.M.M.; Alam, N.M.M.; Silver, B.D.D.; MCGILL, T.J.J.; Tschetter, W.W.W.; Prusky, G.T.T. Independent visual threshold measurements in the two eyes of freely moving rats and mice using a virtual-reality optokinetic system. Vis. Neurosci. 2005, 22, 677-684. [CrossRef] [PubMed]

16. Riepe, R.E.; Norenberg, M.D. Glutamine synthetase in the developing rat retina: An immunohistochemical study. Exp. Eye Res. 1978, 27, 435-444. [CrossRef]

17. Mehalow, A.K.; Kameya, S.; Smith, R.S.; Hawes, N.L.; Denegre, J.M.; Young, J.A.; Bechtold, L.; Haider, N.B.; Tepass, U.; Heckenlively, J.R.; et al. CRB1 is essential for external limiting membrane integrity and photoreceptor morphogenesis in the mammalian retina. Hum. Mol. Genet. 2003, 12, 2179-2189. [CrossRef]

18. van de Pavert, S.A.; Sanz sanz, A.; Aartsen, W.M.; Vos, R.M.; Versteeg, I.; Beck, S.C.; Klooster, J.; Seeliger, M.W.; Wijnholds, J. Crb1 is a determinant of retinal apical muller glia cell features. Glia 2007, 1486-1497. [CrossRef] [PubMed]

19. Quinn, P.M.; Buck, T.M.; Mulder, A.A.; Ohonin, C.; Alves, C.H.; Vos, R.M.; Bialecka, M.; van Herwaarden, T.; van Dijk, E.H.C.; Talib, M.; et al. Human iPSC-derived retinas recapitulate the fetal CRB1 CRB2 complex formation and demonstrate that photoreceptors and müller glia are targets of AAV5. Stem Cell Rep. 2019, 12, 906-919. [CrossRef]

20. McGill, T.J.; Prusky, G.T.; Douglas, R.M.; Yasumura, D.; Matthes, M.T.; Lowe, R.J.; Duncan, J.L.; Yang, H.; Ahern, K.; Daniello, K.M.; et al. Discordant anatomical, electrophysiological, and visual behavioral profiles of retinal degeneration in rat models of retinal degenerative disease. Investig. Ophthalmol. Vis. Sci. 2012, 53, 6232-6244. [CrossRef]

21. Ray, T.A.; Cochran, K.; Kozlowski, C.; Wang, J.; Alexander, G.; Cady, M.A.; Spencer, W.J.; Ruzycki, P.A.; Clark, B.S.; Laeremans, A.; et al. Comprehensive identification of mRNA isoforms reveals the diversity of neural cell-surface molecules with roles in retinal development and disease. Nat. Commun. 2020, 11, 1-20. [CrossRef]

22. Boon, N.; Wijnholds, J.; Pellissier, L.P. Research models and gene augmentation therapy for CRB1 retinal dystrophies. Front. Neurosci. 2020, 14, 1-13. [CrossRef]

23. Berger, A.; Cavallero, S.; Dominguez, E.; Barbe, P.; Simonutti, M.; Sahel, J.A.; Sennlaub, F.; Raoul, W.; Paques, M.; Bemelmans, A.P. Spectral-domain optical coherence tomography of the rodent eye: Highlighting layers of the outer retina using signal averaging and comparison with histology. PLoS ONE 2014, 9, e96494. [CrossRef]

24. Aleman, T.S.; Cideciyan, A.V.; Aguirre, G.K.; Huang, W.C.; Mullins, C.L.; Roman, A.J.; Sumaroka, A.; Olivares, M.B.; Tsai, F.F.; Schwartz, S.B.; et al. Human CRB1-associated retinal degeneration: Comparison with the rd8 Crb1-mutant mouse model. Investig. Ophthalmol. Vis. Sci. 2011, 52, 6898-6910. [CrossRef] [PubMed]

25. Ryals, R.C.; Andrews, M.D.; Datta, S.; Coyner, A.S.; Fischer, C.M.; Wen, Y.; Pennesi, M.E.; McGill, T.J. Long-term characterization of retinal degeneration in royal college of surgeons rats using spectral-domain optical coherence tomography. Investig. Ophthalmol. Vis. Sci. 2017, 58, 1378-1386. [CrossRef]

26. van Rossum, A.G.S.H.; Aartsen, W.M.; Meuleman, J.; Klooster, J.; Malysheva, A.; Versteeg, I.; Arsanto, J.P.; Le Bivic, A.; Wijnholds, J. Pals1/Mpp5 is required for correct localization of $\mathrm{Crb1}$ at the subapical region in polarized Müller glia cells. Hum. Mol. Genet. 2006, 15, 2659-2672. [CrossRef]

27. Pellissier, L.P.; Lundvig, D.M.S.; Tanimoto, N.; Klooster, J.; Vos, R.M.; Richard, F.; Sothilingam, V.; Garrido, M.G.; Bivic, A.L.; Seeliger, M.W.; et al. CRB2 acts as a modifying factor of CRB1-related retinal dystrophies in mice. Hum. Mol. Genet. 2014, 23, 3759-3771. [CrossRef]

28. Quinn, P.M.; Pellissier, L.P.; Wijnholds, J. The CRB1 complex: Following the trail of crumbs to a feasible gene therapy strategy. Front. Neurosci. 2017, 11, 175. [CrossRef]

29. Han, I.C.; Cheng, J.L.; Burnight, E.; Ralston, C.L.; Fick, J.L.; Thomsen, G.J.; Tovar, E.F.; Russell, S.; Sohn, E.H.; Mullins, R.F.; et al. Retinal tropism and transduction of adeno-associated virus (AAV) varies by serotype and route of delivery (intravitreal, subretinal or suprachoroidal) in rats. Hum. Gene Ther. 2020, 1-29. [CrossRef]

30. Charbel Issa, P.; de Silva, S.R.; Lipinski, D.M.; Singh, M.S.; Mouravlev, A.; You, Q.; Barnard, A.R.; Hankins, M.W.; During, M.J.; MacLaren, R.E. Assessment of tropism and effectiveness of new primate-derived hybrid recombinant AAV serotypes in the mouse and primate retina. PLoS ONE 2013, 8, 1-12. [CrossRef]

31. Klimczak, R.R.; Koerber, J.T.; Dalkara, D.; Flannery, J.G.; Schaffer, D.V. A novel adeno-associated viral variant for efficient and selective intravitreal transduction of rat Müller cells. PLoS ONE 2009, 4, e7467. [CrossRef]

32. Kolstad, K.D.; Dalkara, D.; Guerin, K.; Visel, M.; Hoffmann, N.; Schaffer, D.V.; Flannery, J.G. Changes in adeno-associated virus-mediated gene delivery in retinal degeneration. Hum. Gene Ther. 2010, 21, 571-578. [CrossRef] [PubMed]

33. Smith, A.J.; Schlichtenbrede, F.C.; Tschernutter, M.; Bainbridge, J.W.; Thrasher, A.J.; Ali, R.R. AAV-mediated gene transfer slows photoreceptor loss in the RCS rat model of retinitis pigmentosa. Mol. Ther. 2003, 8, 188-195. [CrossRef] 
34. Da Costa, R.; Röger, C.; Segelken, J.; Barben, M.; Grimm, C.; Neidhardt, J. A novel method combining vitreous aspiration and intravitreal AAV2/8 injection results in retina-wide transduction in adult mice. Investig. Ophthalmol. Vis. Sci. 2016, 57, 5326-5334. [CrossRef] [PubMed]

35. Luhmann, U.F.O.; Carvalho, L.S.; Holthaus, S.M.; Cowing, J.A.; Greenaway, S.; Chu, C.J.; Herrmann, P.; Smith, A.J.; Munro, P.M.G.; Potter, P.; et al. The severity of retinal pathology in homozygous Crb1rd8/rd8 mice is dependent on additional genetic factors. Hum. Mol. Genet. 2015, 24, 128-141. [CrossRef]

36. Nishiguchi, K.M.; Carvalho, L.S.; Rizzi, M.; Powell, K.; Holthaus, S.-M.; Azam, S.A.; Duran, Y.; Ribeiro, J.; Luhmann, U.F.O.; Bainbridge, J.W.B.; et al. Gene therapy restores vision in rd1 mice after removal of a confounding mutation in Gpr179. Nat. Commun. 2015, 6, 6006. [CrossRef] [PubMed]

37. Tanimoto, N.; Sothilingam, V.; Kondo, M.; Biel, M.; Humphries, P.; Seeliger, M.W. Electroretinographic assessment of rod- and cone-mediated bipolar cell pathways using flicker stimuli in mice. Sci. Rep. 2015, 5, 1-7. [CrossRef]

38. Tanimoto, N.; Akula, J.D.; Fulton, A.B.; Weber, B.H.F.; Seeliger, M.W. Differentiation of murine models of "negative ERG" by single and repetitive light stimuli. Doc. Ophthalmol. 2016, 132, 101-109. [CrossRef]

39. Alves, C.H.; Wijnholds, J. AAV gene augmentation therapy for CRB1-associated retinitis pigmentosa. In Methods in Molecular Biology (Clifton, N.J.); Springer: Berlin/Heidelberg, Germany, 2018; Volume 1715, pp. 135-151. ISBN 9781493975228.

40. Klooster, J.; Blokker, J.; ten Brink, J.B.; Unmehopa, U.; Fluiter, K.; Bergen, A.A.B.; Kamermans, M. Ultrastructural localization and expression of TRPM1 in the human retina. Investig. Ophthalmol. Vis. Sci. 2011, 52, 8356-8362. [CrossRef]

41. Faas, F.G.A.; Cristina Avramut, M.; van den Berg, B.M.; Mieke Mommaas, A.; Koster, A.J.; Ravelli, R.B.G. Virtual nanoscopy: Generation of ultra-large high resolution electron microscopy maps. J. Cell Biol. 2012, 198, 457-469. [CrossRef] 